\title{
The Western Alboran Gyre: An Analysis of Its Properties and Its Exchange with Surrounding Water 0
}

\author{
Genevieve Jay Brett, ${ }^{a}$ Larry J. Pratt, ${ }^{\mathrm{b}}$ Irina I. Rypina, ${ }^{\mathrm{b}}$ And José C. SÁnchez-Garrido ${ }^{\mathrm{c}}$

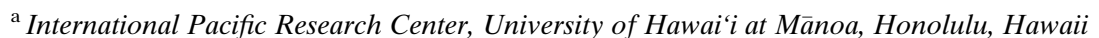 \\ ${ }^{\mathrm{b}}$ Woods Hole Oceanographic Institution, Woods Hole, Massachusetts \\ ${ }^{\mathrm{c}}$ Physical Oceanography Group, Department of Applied Physics II, University of Malaga, Malaga, Spain
}

(Manuscript received 31 January 2020, in final form 12 August 2020)

\begin{abstract}
One of the largest and most persistent features in the Alboran Sea is the Western Alboran Gyre (WAG), an anticyclonic recirculation bounded by the Atlantic Jet (AJ) to the north and the Moroccan coast to the south. Eulerian budgets from several months of a high-resolution model run are used to examine the exchange of water across the Eulerian WAG's boundary and the processes affecting the salinity, temperature, and vorticity of the WAG. The volume transport across the sides of the WAG is found to be related to vertical isopycnal movement at the base of the gyre. Advection is found to drive a decay in the salinity minimum and anticyclonic vorticity of the Eulerian WAG. Given the large contributions of advection, a Lagrangian analysis is performed, revealing geometric aspects of the exchange that are hidden in an Eulerian view. In particular, stable and unstable manifolds identify a stirring region around the outer reaches of the gyre where water is exchanged with the WAG on a time scale of weeks. Its complement is an inner core that expands with depth and exchanges water with its surroundings on much longer time scales. The $3 \mathrm{D}$ evolution of one parcel, or lobe, of water as it enters the WAG is also described, identifying a general Lagrangian subduction pathway.
\end{abstract}

KEYWORDS: Ocean; Mediterranean Sea; Fluxes; Lagrangian circulation/transport; General circulation models

\section{Introduction}

One of the largest and most persistent features in the Alboran Sea, just east of the Strait of Gibraltar in the Mediterranean, is the Western Alboran Gyre (WAG), an anticyclonic recirculation bounded by the Atlantic Jet (AJ) to the north and the Moroccan coast to the south. This feature and its variability have been observed by both in situ and satellite efforts (Peliz et al. 2013; Renault et al. 2012; Heburn and La Violette 1990). The AJ begins at the Strait of Gibraltar as the surface inflow of a reverse estuarine exchange; Mediterranean Water flows westward into the Atlantic below. The WAG has water properties similar to those in the AJ near its edges, with its temperature increasing toward its center, where there is a salinity minimum near 50-m depth (Viúdez et al. 1996).

Dynamical studies have concentrated on the WAG's occasional collapse (Sánchez-Garrido et al. 2013) and reformation (Preller 1986; Whitehead and Miller 1979; Bormans and Garrett 1989). In contrast, this work considers outstanding questions on the processes maintaining the gyre's properties when it is present. These include identification of the drivers of the WAG's warmth compared to its surroundings, its salinity minimum, and its continued anticyclonic rotation. The salinity minimum has been discussed in Viúdez et al. (1996), where it was observed that geostrophic streamlines separate the AJ and WAG and suggested that time-dependent ageostrophic flow

Supplemental information related to this paper is available at the Journals Online website: https://doi.org/10.1175/JPO-D-200028.s1.

Corresponding author: Genevieve Jay Brett, brett33@hawaii.edu must therefore connect the inflow to the salinity minimum. The drivers of the WAG's rotation may include wind stress, lateral forcing from the AJ, or added anticyclonic vorticity from the rising of deep waters as they approach the Strait of Gibraltar, as discussed by Bryden and Stommel (1982).

In this work, we investigate the controls of the WAG's properties by examining Eulerian budgets of volume, temperature, salinity, and vorticity in a mesoscale-resolving numerical model of the Alboran Sea. Our model is the MITgcm (Marshall et al. 1997), run regionally with realistic forcing from reanalyses. The model setup and a comparison to observations is in section 2. The resultant budgets, section 3 , elucidate the contributions of different physical processes, including advection, diffusion, and surface forcing, to the WAG's water properties.

Although the Eulerian approach is traditional and informative, it could be subject to several objections related to the definition of the WAG. Although it is easy to "see" this anticyclonic recirculation in observations and models, an exact boundary can be defined in many ways. Thus, one objection to an Eulerian analysis is that the fixed boundaries of the gyre may depend on the mean flow property (density, salinity, streamlines) one happens to choose: it is likely that a chosen limiting value from each property will provide a different boundary. Another objection is that eddy fluxes across the mean boundary might simply be due to movement of some true (instantaneous) gyre boundary back and forth across the mean boundary. This would be especially problematic if this true gyre underwent net translation in a certain direction over the time period considered, something that does not appear to happen in the present case (see section $2 \mathrm{~b}$ and the online supplemental material for further discussion). These considerations suggest that we instead take a Lagrangian approach in 
which the gyre's boundaries are time dependent and coincide with certain distinguished material curves or surfaces. If the gyre is enclosed by a material contour or surface that remains coherent over time, then one might pick this surface as the boundary, a choice that eliminates eddy fluxes. Several definitions of such distinguished bounding material contours could be used (e.g., Haller and Beron-Vera 2012; Haller 2015; Haller et al. 2016, 2018; Hadjighasem et al. 2017); for example, one could choose a material contour that undergoes minimal stretching. However, the exterior regions of the anticyclonic recirculation that one intuitively still qualifies as the WAG experience significant exchange of material with their surroundings, and thus all material contours in this region undergo significant stretching. This means that a minimal-stretching contour definition would be quite restrictive, likely indicating a region much smaller than the full extent of the anticyclonic recirculation. To capture the larger anticyclonic gyre, we therefore define a time-dependent boundary that permits material exchange between the gyre and its surroundings. In section 4 , we employ the method of lobe analysis (e.g., Samelson 1992; Duan and Wiggins 1996; Coulliette and Wiggins 2000; Deese et al. 2002; Mancho et al. 2003; Yuan et al. 2004; Rypina et al. 2009, 2010; summarized by Samelson and Wiggins 2006) to identify one such boundary. Miller et al. (2002) successfully employed this method to analyze a more idealized, aperiodic, boundarytrapped gyre in a 2D flow. We extend the analysis to the threedimensional, time-varying WAG, using maps of manifolds to distinguish geographical regions near the periphery of the WAG that are more likely to exchange fluid with surroundings on a time scale of two weeks, and we describe the exchange through the turnstile lobe mechanism. This analysis will lead to insight into the kinematics, geographical extent, and time scales of exchange between the gyre and its surroundings, and will also show how fluid subducts or upwells along isopycnal surfaces as it is moved into or out of the gyre.

We emphasize, however, that the Lagrangian approach is not a cure-all. It is computationally difficult to implement, especially in three dimensions (e.g., Branicki and Kirwan 2010), and the small scales that arise due to the stretching and folding of the material surfaces make it extremely challenging to compute the content of the lobes and to close property budgets for the Lagrangian gyre as a whole. In addition, lobe dynamics applied to time-aperiodic flow fields requires intuitive choices about how to configure the boundary (Haller and Poje 1998; Miller et al. 2002). In the end, we have to recognize that the Eulerian and Lagrangian approaches yield different information and that, in the case of the WAG, neither approach is superior or unambiguous. This work does not attempt to resolve this ambiguity: instead, we simply present calculations based on one reasonable choice of a fixed gyre boundary and one reasonable choice of a moving boundary.

\section{Model description}

\section{a. Model setup}

We use the MITgcm in hydrostatic mode (Marshall et al. 1997), configured similarly to Sánchez-Garrido et al. (2013) but with increased horizontal resolution in the western Alboran. This model solves the Boussinesq form of the Navier-Stokes equations for an incompressible fluid with a finite-volume spatial discretization on a curvilinear grid with typical horizontal resolution of $1-3 \mathrm{~km}$ (see Fig. 1). The vertical grid has 46 layers with $5-\mathrm{m}$ resolution at the surface, decreasing with depth to $403 \mathrm{~m}$ at a maximum depth of $4288 \mathrm{~m}$. The model domain includes the Strait of Gibraltar and has open boundaries in the Atlantic and Mediterranean.

Surface forcings are the ERA-Interim reanalysis daily heat and salt fluxes at $0.75^{\circ} \times 0.75^{\circ}$ resolution and the IFREMER CERSAT (Institut Français de Recherche pour l'Exploitation de la Mer Centre ERS d'Archivage et de Traitement) 6-hourly $10-\mathrm{m}$ global blended mean wind fields at $1 / 4^{\circ}$. Open east and west ocean boundaries are forced with daily $1 / 12^{\circ}$ velocity, salinity, and temperature from the Atlantic-Iberian Biscay IrishOcean Physics Reanalysis (AIBIOPR) with a sponge layer of $80 \mathrm{~km}$ width and a $1-\mathrm{h}$ relaxation time scale.

Initial conditions for temperature and salinity are from the 16-28 September 2011 model output from Sánchez-Garrido et al. (2013), but averaged over the two weeks and the latitudinal extent of the basin, leaving only east-west and vertical gradients; this smoothing allowed a more stable circulation to develop. The model was spun up for 55 simulated days without atmospheric forcing and with constant fall 2007 average ocean boundary conditions. Over 75 further simulated days, mean fall 2007 atmospheric forcing was added, followed by a transition to mean October and then November forcing for both the atmosphere and ocean boundaries. Each of these transitions was a linear temporal interpolation of the forcings and boundary conditions over five days, with twenty days of the full forcing in each case. After this total of 130 days spinup, the fully forced simulation began running from 1 November 2007. The model run ends 31 December 2008.

\section{b. Comparison to observations}

This MITgcm configuration is run without any restoring term toward or assimilation of observations; therefore, we expect it to deviate from observations over time. Although simulations utilizing data assimilation might better match observations, the process of nudging toward observations would interfere with the budget calculations we wish to perform. We compare model output to observations not as a formal validation of the model but in order to identify the time period when using the model to study a stable WAG is feasible. As a primary check, the MITgcm output daily-mean sea surface height (SSH) is compared to satellite-based gridded daily fields from AVISO (distributed by Copernicus Marine Environmental Monitoring Service). These gridded fields are the best continuous observations available of the circulation, but are based on only 2-3 daily satellite tracks. When patterns of SSH match, near-surface geostrophic flows are similar; we use this comparison to identify when the WAG is stable and of the correct sign, size, and position. Secondarily, we compare the outflow at the western end of the Strait of Gibraltar from the model and from ADCP measurements to check that the exchange between the Atlantic and the Mediterranean is of the correct magnitude and structure. 

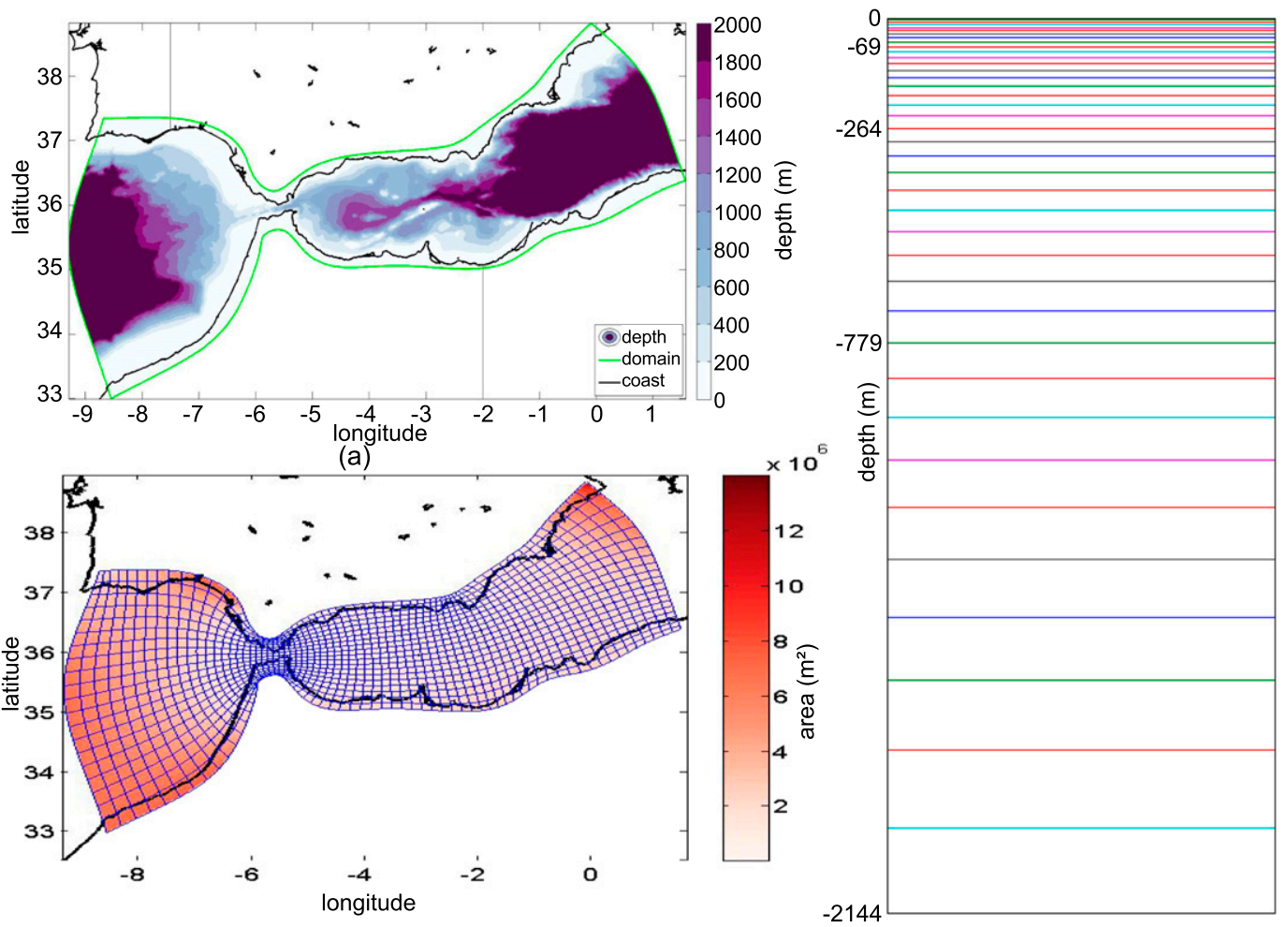

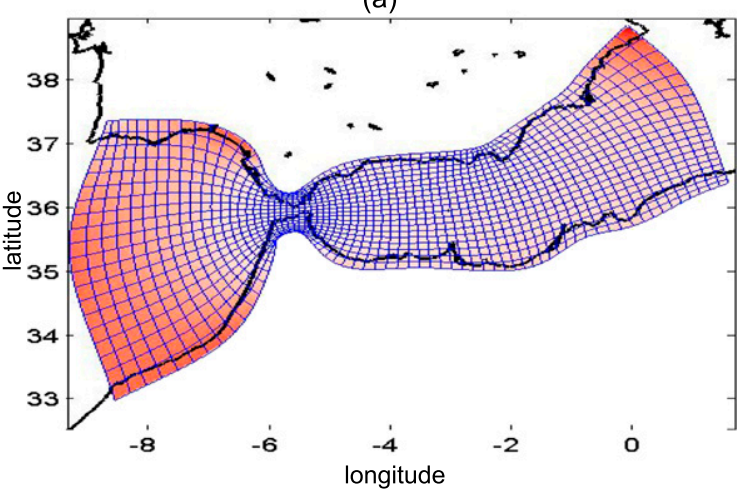

(b)

(c)

FIG. 1. (a) Domain of the simulation, edges in green. Color is bathymetric depth, and black curves are the coast. Black lines on land indicate approximate borders between Portugal and Spain (northern) and Morocco and Algeria (southern). (b) Model grid, with every tenth gridline in blue over the area of each cell in red scale $\left(\mathrm{m}^{2}\right)$ and the coast indicated in black. (c) Depth grid spacing in meters, top 40 interfaces, with interfaces 1, 10, 20, 30, and 40 labeled. Interfaces $41-46$ are at $2368,2615,2888,3189,3520$, and $3885 \mathrm{~m}$.

Monthly mean maps of SSH illustrate the broad patterns of the circulation (Fig. 2). The first five and final two months of the run show a clear WAG as a positive anomaly in a consistent position. In the intervening months, the AVISO SSH continues to show the WAG, with the addition of a developing and then decaying Eastern Alboran Gyre. In contrast, the model SSH shows the loss of the WAG, with the whole basin having low $\mathrm{SSH}$ in the interior, indicating an overall cyclonic circulation pattern that is not frequently observed; April is representative of these months (Fig. 2). These intervening months are an example of the coastal mode of circulation that has been observed for shorter periods in satellite SST, including June 1986 (Heburn and La Violette 1990) and February 1998 (VargasYáñez et al. 2002). Heburn and La Violette (1990) found a similarly prolonged cyclonic circulation for a one active layer reduced gravity model forced by a seasonal cycle of climatological winds. An anticyclonic gyre begins to redevelop in our model run in July, and reaches the usual position and size of the WAG in December. We are interested in analyzing a period when the WAG is present and stable over an extended period, the first five months of the model run being a good candidate. To identify the end of that period, we computed the spatial correlation coefficient, $r$, between the daily AVISO SSH maps and the daily model SSH fields interpolated to the lowerresolution AVISO grid for both the full Alboran basin and the section west of $2.3^{\circ} \mathrm{W}$, which typically contains the WAG (Fig. 3a). The correlation coefficient in the western Alboran Sea is near 0.6 during the first 148 days and this is regarded as satisfactory agreement. There is a sharp drop in $r$ after 148 days, near the end of March, which is consistent with April being the first month where the model SSH is very different from the observations. Based on the SSH comparisons, we chose the first 148 days, November 2007-March 2008, for the model analysis. This analysis period ends 9 days before the daily SSH field shows the loss of the WAG.

The mean SSH during these 148 days shows broad agreement between model and satellite (Fig. 3b). As with individual months, the broad patterns are similar, with a clear WAG centered near $4^{\circ} \mathrm{W}$, although the modeled WAG is more circular and more separated from the northern coast. East of the WAG, there are two cyclonic features, one near $2^{\circ} \mathrm{W}$, which is stronger in observations, and one near $0^{\circ} \mathrm{W}$, which is larger and stronger in the model. The model SSH standard deviation patterns (Fig. 3c) are much less similar to those observed. Both have a matching high in the east centered around $37^{\circ} \mathrm{N}, 0^{\circ} \mathrm{W}$, with an elevated variance "tail" extending southwest from the high. In the Western Alboran region, however, observations show higher variance near the WAG's center, while the model shows higher variance on the WAG's eastern edge where the AJ turns south. In AVISO, 

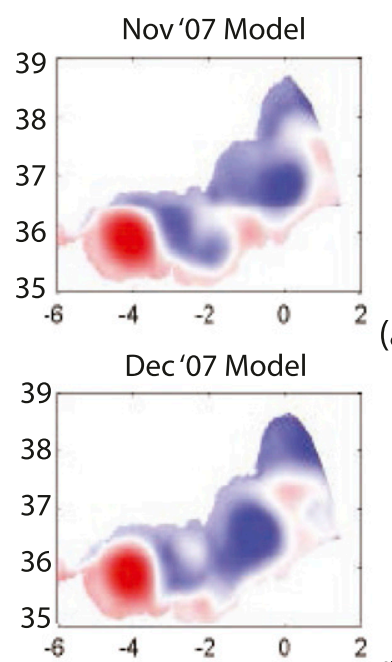

(a)
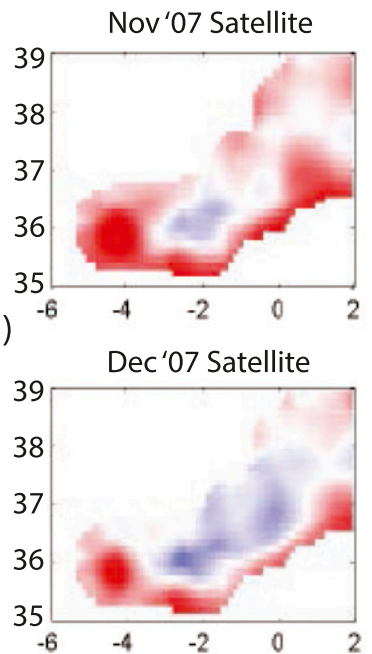

(b)
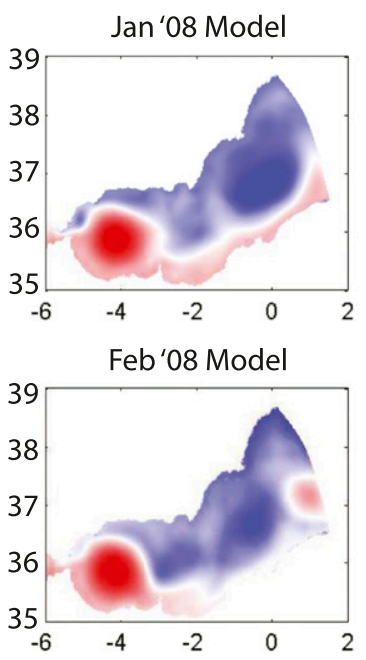

(d)
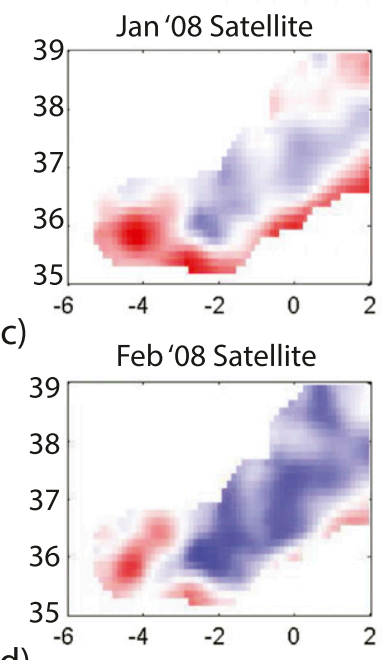
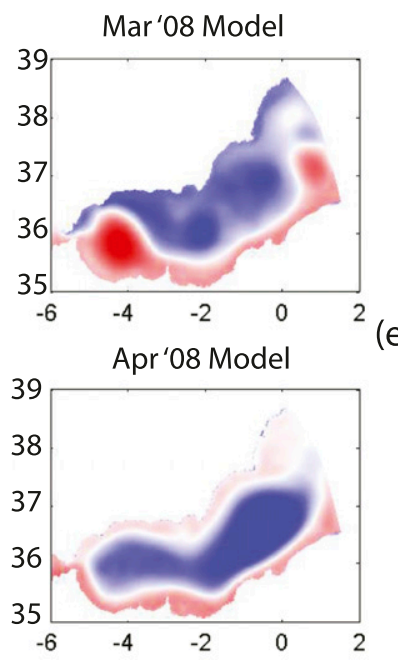

(e)
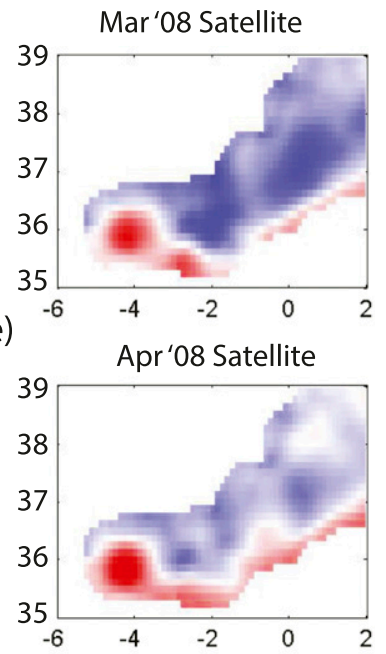

(f)
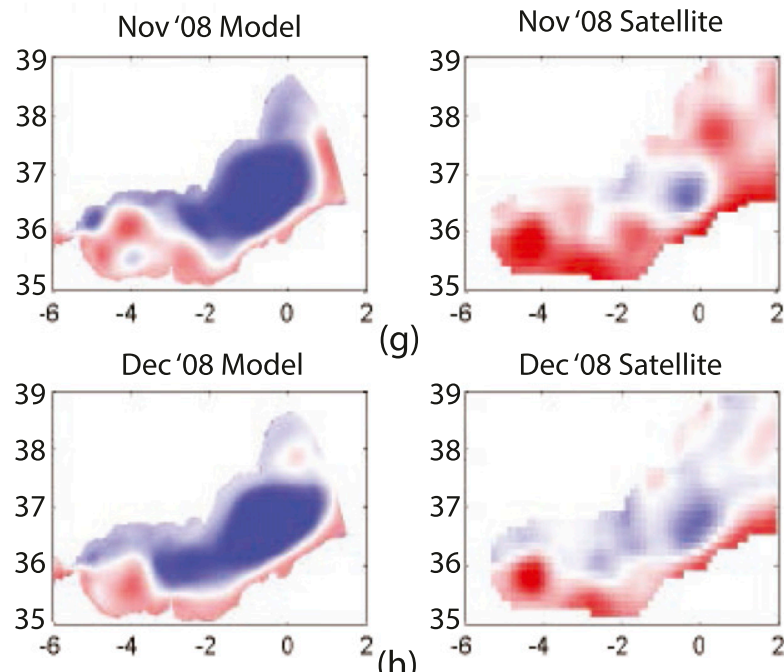

${ }^{2}(\mathrm{~h})$

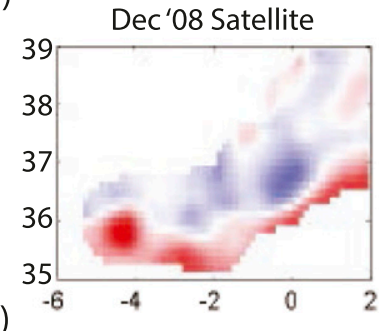

FIG. 2. Monthly mean SSH (saturated colors at $0.2 \mathrm{~m}$ in red and $-0.2 \mathrm{~m}$ in blue), comparison between MITgcm output and AVISO data. November 2007-April 2008 and November-December 2008. Additional months available in Brett (2018).

the WAG varies in strength (e.g., SSH anomaly) more than position during this time, and it remains beyond the period analyzed, while in the model, the WAG varies more in eastward extent than strength, and it decays after the period analyzed.

As a consistency check, we also compared the structure and magnitude of the inflow/outflow at the Strait of Gibraltar between the model and observations. In the model, both the eastward inflow connecting to the AJ and the westward outflow at depth are on the order of $1 \mathrm{~Sv}\left(1 \mathrm{~Sv} \equiv 10^{6} \mathrm{~m}^{3} \mathrm{~s}^{-1}\right)$. Sammartino et al. (2015) computed the westward flux using 10 years of velocity data from a mooring on the western end of the Strait. Generally this outflow is between 0.75 and $0.9 \mathrm{~Sv}$, with a seasonal cycle that peaks in the spring. The interface depth, between the inflow and outflow, is below $190 \mathrm{~m}$. Our model shows the correct structure of inflow at the top and outflow below, with a mean of $1.1 \mathrm{~Sv}$ for both, which is of the correct magnitude. The interface depth is higher in the model than observations, most often $163 \mathrm{~m}$, which is within 1-2 grid cells of the observed depth.

\section{c. Mean Western Alboran Gyre}

Before analyzing the WAG, we further describe its mean state during the 148-day period under examination, when the gyre's position and shape match observations reasonably well. At the surface, we show the relative vorticity, horizontal velocity, and wind stress (Fig. 4), which indicate that the WAG is generally anticyclonic, as expected, with an overall westward wind stress. The horizontal extent of the Eulerian WAG is chosen to include surface grid cells with a mean practical salinity below 36.475 , which is nearly equivalent to a mean potential density limit of $\sigma_{\theta}=26.3 \mathrm{~kg} \mathrm{~m}^{-3}$ or a mean SSH above $0.05 \mathrm{~m}$. This value is chosen in order to include the full extent of the WAG from the southeastern edge of the Strait of Gibraltar (Ceuta) to Cabo Tres Forcas, the cape at about $3^{\circ} \mathrm{W}$, without continuing farther east in the AJ. This choice of an Eulerian 


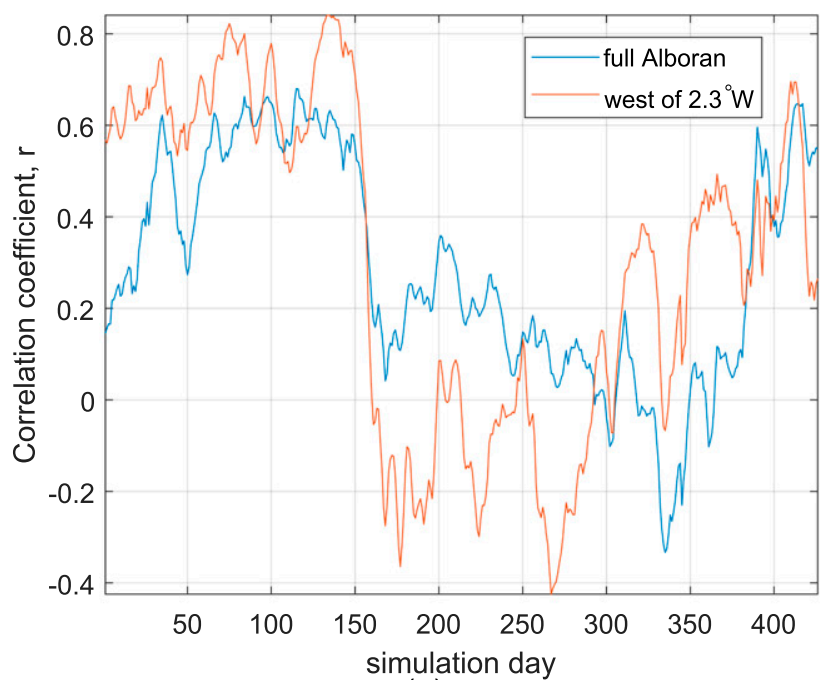

(a)
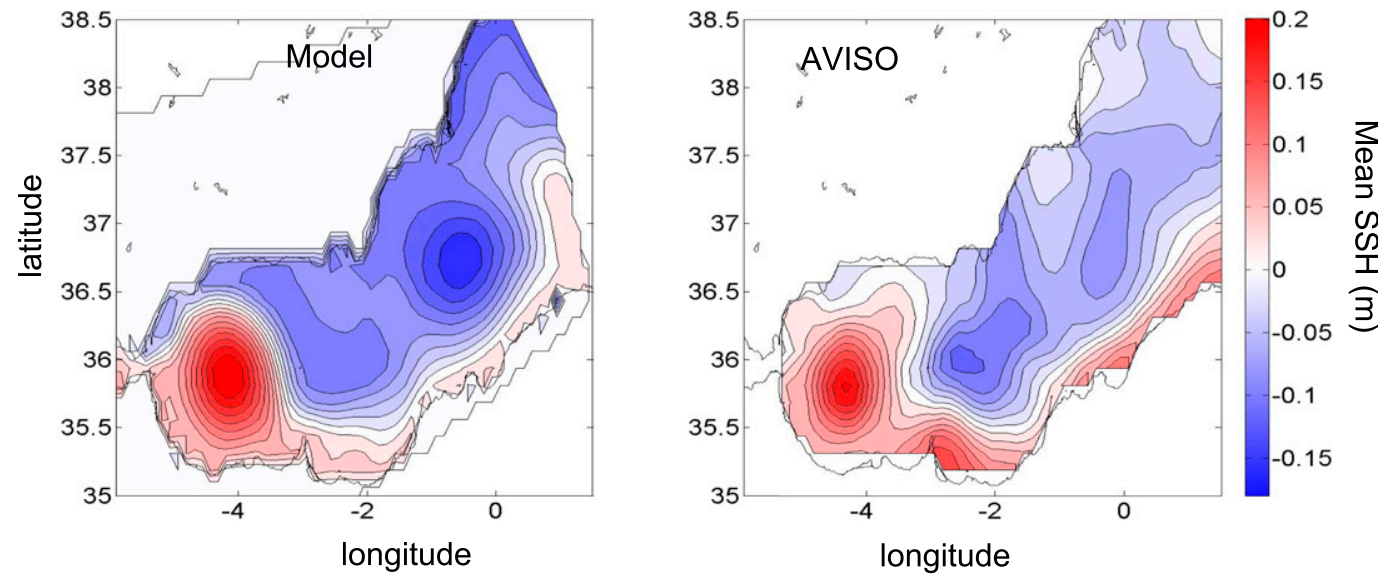

(b)
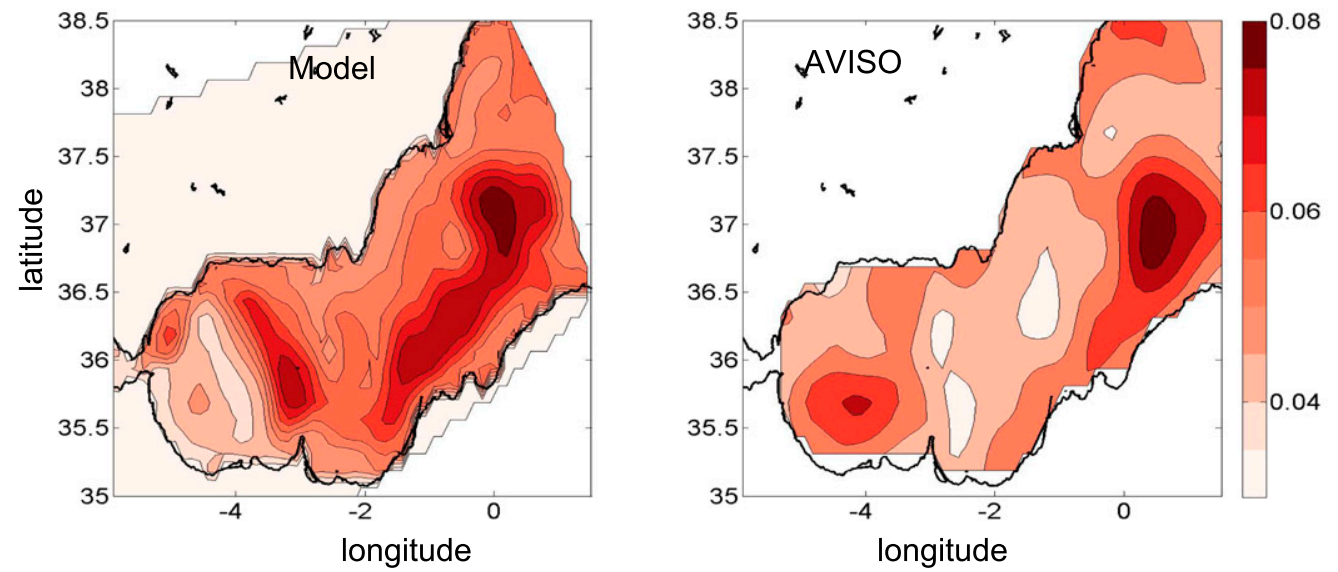

FIG. 3. (a) Daily SSH correlation coefficient $r$ between output and observations for all points (blue) and points west of $2.3^{\circ} \mathrm{W}$ (orange) each day of the simulation, where day 1 is 1 Nov 2007. (b) Mean SSH (m) over the 148-day well-correlated period, comparison between (left) model output and (right) AVISO data. (c) Standard deviation of daily SSH (m) over the same period. 


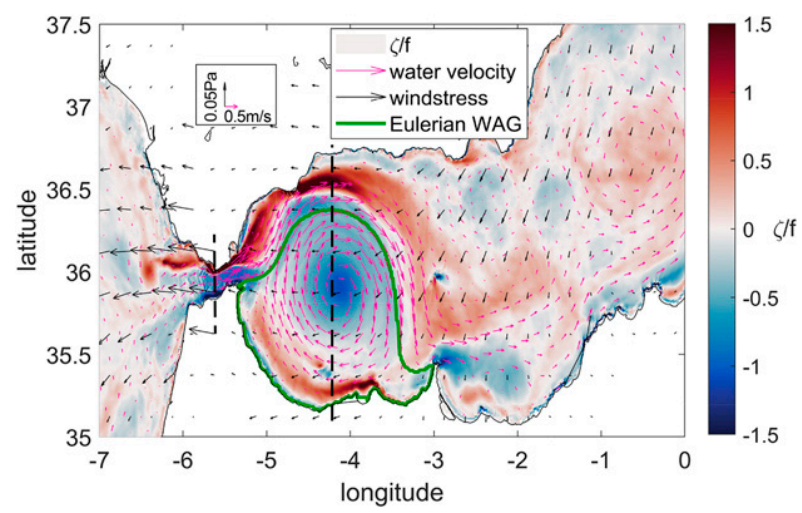

FIG. 4. Mean surface relative vorticity normalized by planetary vorticity $\zeta / f$ in color. Magenta vectors show the mean surface water velocity, with a scale of $0.5 \mathrm{~m} \mathrm{~s}^{-1}$ shown in the box near $\left(37^{\circ}\right.$, $\left.-5.5^{\circ}\right)$. Black vectors show the mean wind stress, with a scale of $0.05 \mathrm{~Pa}$ shown in the box near $\left(37^{\circ},-5.5^{\circ}\right)$. The green curve shows the chosen Eulerian WAG horizontal boundary. Black dashed lines indicate the locations of sections shown in Fig. 5.

boundary is somewhat subjective, but examinations of monthly maps of surface features indicate there are no large excursions or sustained directional migrations of the dynamic gyre across this fixed boundary (additional discussion in the online supplemental material). A sensitivity analysis with a slightly modified Eulerian WAG boundary showed that all qualitative results are insensitive to small changes (details in the online supplemental material).

We show meridional sections of the water properties in the WAG and the Strait of Gibraltar to describe their vertical structure (locations in Fig. 4, sections in Fig. 5). The zonal velocity with overlaid $\sigma_{\theta}$ contours in the Strait (Fig. 5a) shows that the inflow is typically above the $\sigma_{\theta}=27.5 \mathrm{~kg} \mathrm{~m}^{-3}$ contour and stronger on the northern side. All potential densities discussed are surface-referenced density anomalies $\sigma_{\theta} ; \sigma_{\theta}=$ $27 \mathrm{~kg} \mathrm{~m}^{-3}$, for instance, indicates a surface-referenced potential density of $1027 \mathrm{~kg} \mathrm{~m}^{-3}$. The WAG's velocities get weaker with depth and the center shifts northward near 200-m depth (Fig. 5b). This latitudinal shift near the $\sigma_{\theta}=28$ contour is related to the addition of westward flow along the African shore connected to the outflow through the Strait of Gibraltar (as described in Bryden and Stommel 1982). Because we are interested in the exchange between the WAG and the AJ, our analysis focuses on near-surface flow where the AJ is evident. Specifically, we consider the part of the water column between the ocean surface and the $\sigma_{\theta}=27.5$ potential density contour, which includes the less-stratified surface layer above $\sigma_{\theta}=$ $26.5 \mathrm{~kg} \mathrm{~m}^{-3}$ (50-100 $\mathrm{m}$ deep) and the top of the pycnocline between $\sigma_{\theta}=26.5$ and $27.5 \mathrm{~kg} \mathrm{~m}^{-3}$.

Sections of potential temperature with salinity contours (Figs. 5c,d) show similar structures to the density. All salinity values are on the practical salinity scale, which is dimensionless. The differences include subsurface salinity minima in the center of the WAG (Fig. 5d) and southern side of the Strait (Fig. 5c). These minima are consistent with descriptions from observations (Viúdez et al. 1996). Overall, the
Eulerian WAG is warmer and fresher than waters below it or to the north.

\section{Eulerian analysis}

In this section, we quantify changes in the average hydrographic and dynamical properties of the WAG and identify the processes responsible using Eulerian budgets comprised of the terms in the model's governing equations. First, a volume budget allows us to quantify the rate of exchange of water between the Eulerian WAG and its surroundings. Next (section 3a), we address the maintenance of the relatively warmer and fresher waters of the Eulerian WAG through heat and salt budgets. Finally, in section $3 b$, a vorticity budget identifies the processes that support the anticyclonic rotation.

As stated in the previous section, the Eulerian WAG in our analysis is limited in the horizontal to those cells with a mean surface salinity less than 36.475 and in the vertical to cells with mean potential density less than $\sigma_{\theta}=27.5 \mathrm{~kg} \mathrm{~m}^{-3}$. Thus, the Eulerian WAG boundary consists of a vertical cylinder extending from the surface $S=36.475$ contour down to the mean $\sigma_{\theta}=27.5 \mathrm{~kg} \mathrm{~m}^{-3}$ surface. This gives a fixed control volume consisting of whole grid cells.

A volume budget is computed with four terms, which may be written

$$
\frac{d V_{\mathrm{SSH}}}{d t}=-\int_{\text {sides }} \mathbf{u} \cdot d \mathbf{A}-\int_{\sigma} \mathbf{u} \cdot d \mathbf{A}-\int_{\text {surface }} F_{\mathrm{PR}} d A,
$$

where $d \mathbf{A}$ is the differential area vector perpendicular to the boundary and positive outward, the integral over the sides gathers horizontal fluxes, that over $\sigma$ is across the mean $\sigma_{\theta}=$ 27.5 surface, and that over the surface is across the air-sea interface. Parameter $V_{\mathrm{SSH}}$ is the total volume, whose changes are due to changes in the sea surface relative to mean sea level, and $F_{\mathrm{PR}}$ is the forcing of volume changes by precipitation and evaporation. The term on the left is the change in volume storage due to changes in sea surface height, the first term on the right is net flux of horizontal advection inward through cell sides, the second term on the right is the net flux of advection upward through the bottom, including the sides of the deepest cells, and the final term is precipitation and evaporation at the surface. The budget computation uses daily mean velocities, sea surface height, and surface evaporation and precipitation. After computing all four terms independently from the model output, the budget closes with the sum of all terms being less than $6 \%$ of the smallest contributing term (which is the surface forcing term as will become evident from Fig. 6); this inaccuracy stems in part from using daily-mean SSH to compute the changes in volume.

The primary balance in the Eulerian WAG volume budget is between advection through the sides and that through the bottom, with changes in storage and exchange with the atmosphere being two orders of magnitude smaller (time series in Fig. 6, mean values in Table 1). These advective fluxes are typically on the order of $10^{5} \mathrm{~m}^{3} \mathrm{~s}^{-1}$ but can at times be nearly as large as the $\mathrm{AJ}$ (about $1 \mathrm{~Sv}$ ) or one-third the recirculation of the WAG $\left(2-3 \mathrm{~Sv}\right.$ above $\left.\sigma_{\theta}=27.5\right)$, and change signs frequently but without any clear dominant frequency (spectra were smooth, decreasing with increasing frequency). 


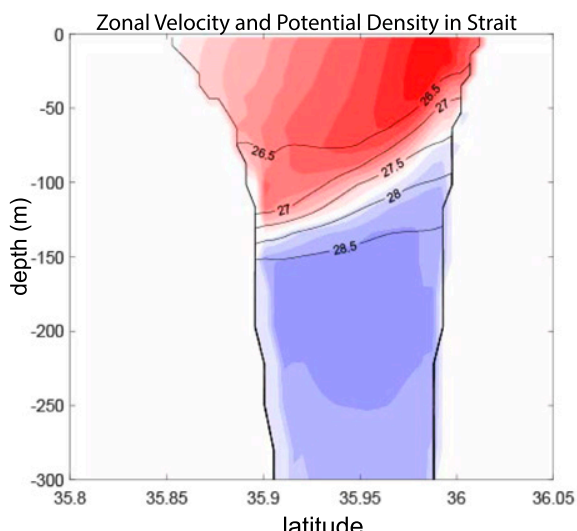

(a)

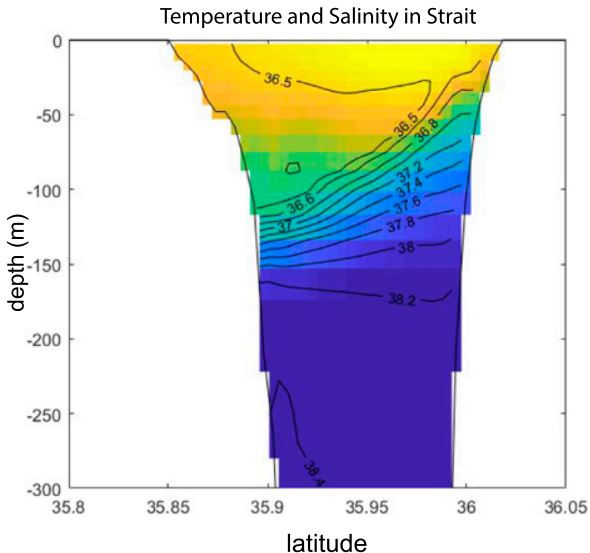

(c)

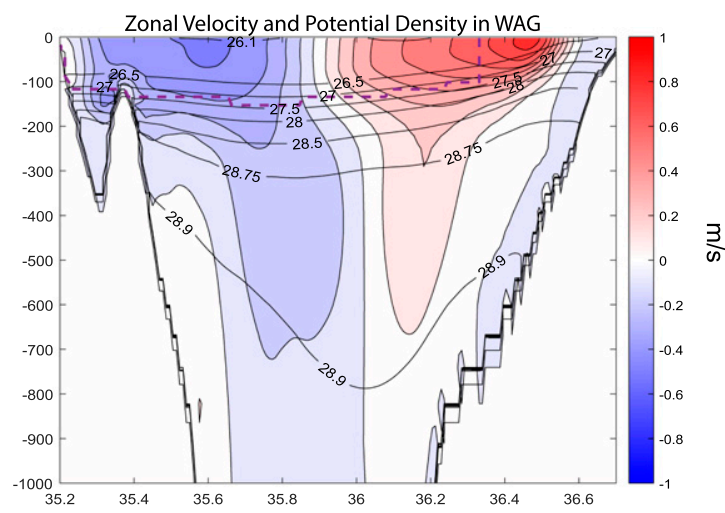

(b)

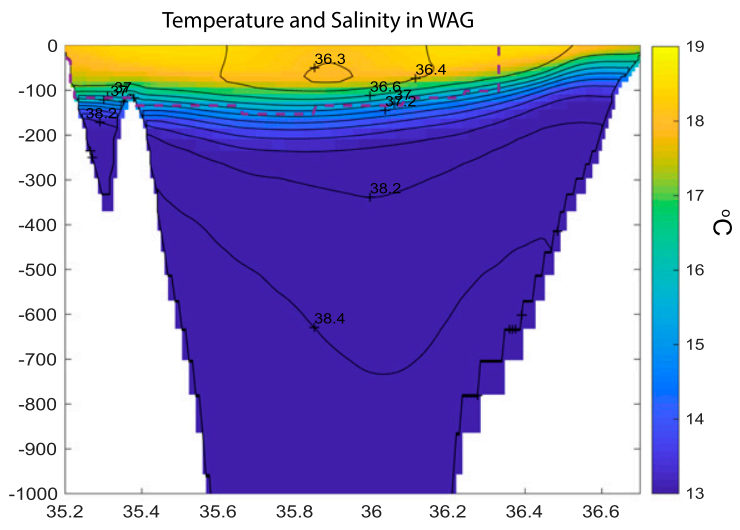

(d)

FIG. 5. Two north-south sections, facing west, of the 148-day mean water properties. (a),(b) Zonal velocity fields with mean potential density anomaly contours $\sigma_{\theta}$ overlaid. (c),(d) Mean potential temperature fields with mean practical salinity contours overlaid. (left) The Strait of Gibraltar. (right) The Western Alboran Gyre, with purple dashed curves marking the chosen extent of the Eulerian WAG.

The horizontal velocity is nearly always parallel to the gyre boundary along the northern edge of the Eulerian WAG, with the time- and contour-averaged normal velocity components being $9 \%$ of the total velocity. The spatial pattern of the timemean normal component of advective fluxes (arrows in Fig. 7) shows that this component of the horizontal advection changes sign along the northwestern arc and is consistently inward along the northeastern arc, with the largest flux through the easternmost segment, which corresponds to the AJ. These patterns, however, vary somewhat over the analysis period, likely related to some shifts in the position of the Lagrangian WAG across our Eulerian boundary (see the online supplemental material for monthly maps). The total magnitude of the horizontal advection through the sides, which is the integral of the absolute value of horizontal advective fluxes, is $8 \times 10^{6} \mathrm{~m}^{3} \mathrm{~s}^{-1}$ in the mean, 20 times larger than the net flux, which is the integral of the horizontal advective fluxes and $4 \times 10^{5} \mathrm{~m}^{3} \mathrm{~s}^{-1}$ in the mean. This disparity between the total volume of water crossing the Eulerian WAG boundary and the net flux indicates that water is mostly flushing through the Eulerian control volume. This includes contributions from both the motion of the Lagrangian
WAG relative to the Eulerian WAG and the exchange of water between the Lagrangian WAG and surroundings. The mean vertical advection through the bottom shows upwelling in the northeast, although this is too far from the coast to be related to coastal upwelling, and high but highly variable in sign values near seamounts, where there is strong temporal variability.

The net advective volume transports, which so nearly oppose each other across the sides and bottom, may be largely due to the heaving of isopycnals. A comparison between the volume flux through the bottom of the Eulerian WAG control volume and the mean velocity of the $\sigma_{\theta}=27.5 \mathrm{~kg} \mathrm{~m}^{-3}$ surface match well, with a correlation coefficient of $r=0.74$ (Fig. 8). When this isopycnal moves up, water flows in through the fixed Eulerian WAG bottom. This water must either continue upward, raising the sea surface height, or spread in the horizontal; since there are no corresponding large changes in the sea surface height, but there are similar horizontal advective fluxes, we conclude that the water spreads horizontally, outward across the Eulerian WAG boundary. In the opposite case, when there is convergence across the sides, there is outflow through the bottom and the associated isopycnal deepens. 


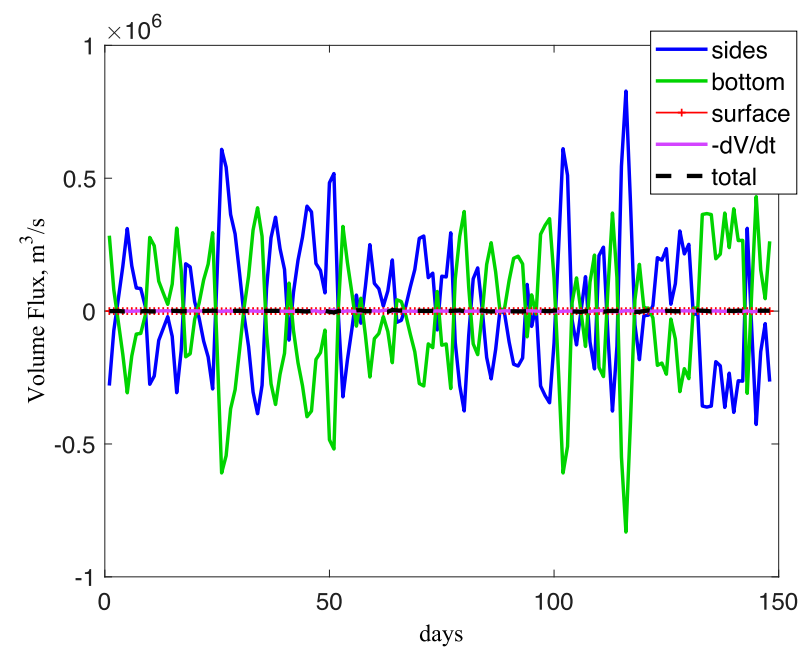

FIG. 6. Total Eulerian WAG volume budget $\left(\mathrm{m}^{3} \mathrm{~s}^{-1}\right)$. These are the net volume transports through the sides (dark blue), bottom (green), and top (red), the volume transport from the change in storage due to changing SSH (purple), and the total of these transports (black dashed).

Thus, a large proportion of the daily advective fluxes is related to the heaving of the $\sigma_{\theta}=27.5 \mathrm{~kg} \mathrm{~m}^{-3}$ surface.

To summarize, our findings from the volume budget are as follows. We formed a volume budget for the Eulerian WAG to understand the exchange of water with its surroundings, which occurs as advection through the sides and bottom. The daily rate of net transport through the sides is typically $10^{5} \mathrm{~m}^{3} \mathrm{~s}^{-1}$, which is balanced primarily by advection through the bottom associated with movement of the $\sigma_{\theta}=27.5 \mathrm{~kg} \mathrm{~m}^{-3}$ surface. This heaving motion indicates that the movement of a Lagrangian WAG, bounded by the instantaneous $\sigma_{\theta}=27.5 \mathrm{~kg} \mathrm{~m}^{-3}$ surface, across our Eulerian WAG boundaries is contributing to the daily volume fluxes. From these analyses we can conclude that water is consistently exchanged between the Eulerian WAG and its surroundings, but further analysis is needed to understand the exchange of properties (sections $3 \mathrm{a}$ and $3 \mathrm{~b}$ ) and the spatial extent of that exchange (section 4).

\section{a. Heat and salt}

To understand the warmth and freshness of the Eulerian WAG, we now compute heat and salt budgets for the same control volume as the volume budget. The concentration budget for any tracer includes changes in storage, advective transports across the sides and bottom, diffusive transports across the sides and bottom, and surface forcing. The MITgcm is Boussinesq and the conservation statements for either temperature or salinity may be written

$$
\begin{aligned}
\frac{d}{d t} \int c d V= & -\int_{\text {side }} c \mathbf{u} \cdot d \mathbf{A}-\int_{\sigma} c \mathbf{u} \cdot d \mathbf{A}+\int_{\text {side }} \kappa_{h} \nabla_{h} c \cdot d \mathbf{A} \\
& +\int_{\sigma} \kappa \nabla c \cdot d \mathbf{A}-\int_{\text {surface }} \mathbf{F} \cdot d \mathbf{A},
\end{aligned}
$$

where each term is in the order stated above; $c$ is either salinity or potential temperature, $\mathbf{u}$ is the three-dimensional velocity
TABLE 1. Time-mean volume transports across the Eulerian WAG boundaries $\left(\mathrm{m}^{3} \mathrm{~s}^{-1}\right.$; positive into the gyre, rounded to the nearest tenth of $\left.\mathrm{a} \mathrm{m}^{3} \mathrm{~s}^{-1}\right)$.

\begin{tabular}{lr}
\hline \multicolumn{1}{c}{ Volume transport } & Mean value \\
\hline Advection through sides & 24953.1 \\
Advection through bottom & -24904.1 \\
Precipitation - evaporation & -7.0 \\
Change in storage via SSH & -41.8 \\
\hline
\end{tabular}

vector, $\kappa$ is the diffusivity including that from turbulence closure, $d \mathbf{A}$ is the differential area vector perpendicular to the boundary and positive outward, and $\mathbf{F}$ is surface forcing, including changes from evaporation and precipitation. Each term is an integral over the appropriate surface, with the sides being vertical, and is translated into absolute transports rather than concentrations using density and specific heat, which is constant; details of computation from model diagnostics are available in Chakraborty and Campin (2014). Finally, the budgets are referenced to the mean value for the control volume during the 148-day period of consideration. After computing each term independently from the model output, the budgets close with the sum of all terms being less than $2 \%$ of the smallest term at all times.

The heat budget, referenced to a 148-day mean Eulerian WAG temperature of $16.99^{\circ} \mathrm{C}$, has changes in storage and advection through the sides and bottom as its largest daily terms (Fig. 9a). Surface forcing is smaller, but its peaks can be as large as advection through the sides or bottom, while diffusion through the bottom is much smaller and diffusion through the sides is negligible. Even though advection through the sides is often partially compensated by advection through the bottom, their sum-total advection-is still significantly larger than the diffusive tracer fluxes or the surface forcing terms (Fig. 9b). In the mean (Fig. 9c), the Eulerian WAG is cooling over time $(d H / d t<0)$, with advection and, to a smaller degree, diffusion through the bottom contributing to this cooling while surface forcing and advection through the sides oppose it. However, monthly means (colored dots in Fig. 9c) show varied signs for all terms except vertical diffusion, with the mean cooling mainly due to February. Cumulative change in heat content and cumulative advective flux (Fig. 9d) change sign twice (and also once come close to zero without changing sign) during the 148-day analysis period. What we can conclude is that the main driver of the changes in heat content, both short term and long term, is the advective flux, which is significantly larger than the surface forcing term, itself significantly larger than the diffusive terms.

Although both the mean temperature during our analysis period and observations of the WAG indicate a temperature maximum in the surface WAG, the large cooling episode driven by horizontal advection calls into question whether that maximum is being supported by ongoing processes. Although our study focuses on the period when WAG is stable and not on its collapse and reformation, it is interesting to note that not long after the end of our period of analysis, the modeled WAG collapses. These collapse and reformation events are typically 


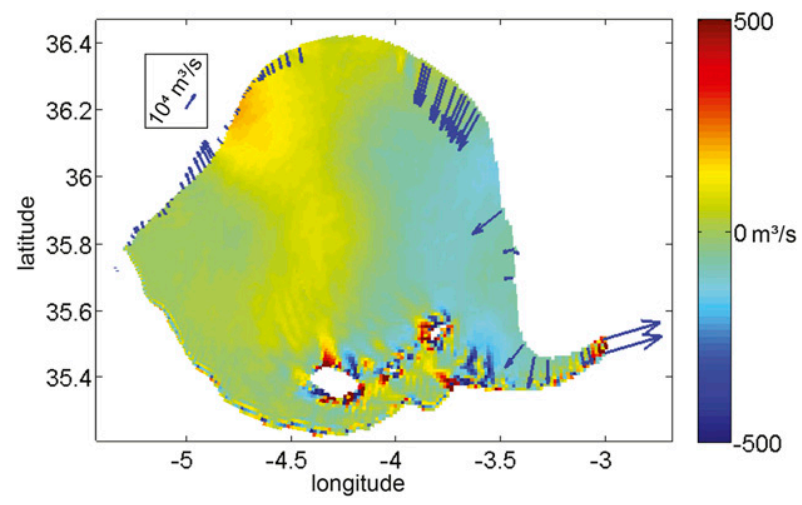

FIG. 7. Mean advective volume transports for the Eulerian WAG. Colors show transport through the bottom, arrows show vertically integrated transport perpendicular to the sides. the topleft arrow shows scale, $1 \times 10^{4} \mathrm{~m}^{3} \mathrm{~s}^{-1}$. White patches indicate seamounts.

observed less than once per year; we know of four welldocumented cases (Viúdez et al. 1998; Vargas-Yáñez et al. 2002; Flexas et al. 2006; Sánchez-Garrido et al. 2013). However, some earlier observations indicate more frequent collapses: in satellite observations from 1982 and 1985-86, Heburn and La Violette (1990) list 5 instances where the WAG is present in one month and absent the next. In our model run, after the collapse of the WAG near day 155, it is reformed during days 240-265. We speculate that the temperature maximum within the WAG is partially due to its formation. During the reformation of the model WAG, the temperature of the AJ, which is now attached to the Moroccan coastline, and that of the forming gyre are higher than waters to the north or east (Figs. 10a-c), supporting the possibility of the spatial temperature maximum being set at formation.

The salt budget (Fig. 11a) for the Eulerian WAG follows the same qualitative pattern as the heat budget: advection through the sides and bottom and the change in salt storage are the largest terms in a daily budget referenced to the mean salinity of 36.61, and all change signs on time scales of days to weeks. The total advection is highly correlated with changes in salt content over time (Fig. 11b), as for the heat. The time-mean budget, Fig. 11c, shows the same dominance of advection as for heat, with slightly larger vertical diffusion and smaller surface forcing. The changes in salt storage always show increasing salt when averaged each month (colored dots in Fig. 11c), in contrast to the changes in heat storage. Cumulative fluxes (Fig. 11d) show that the time-integrated total advective flux is almost always positive, with a mostly positive slope. These qualities indicate that total advection adds salt to the comparatively fresh Eulerian WAG on time scales of weeks to months despite its daily fluctuations in sign.

The salinity value at its minimum within the WAG is matched only by the minimum salinity value in the southern subsurface Strait of Gibraltar. Viúdez et al. (1996) hypothesized that the connection of the salinity minimum in the WAG to the Strait of Gibraltar is through a time-dependent advection across the observed mean geostrophic streamfunction.

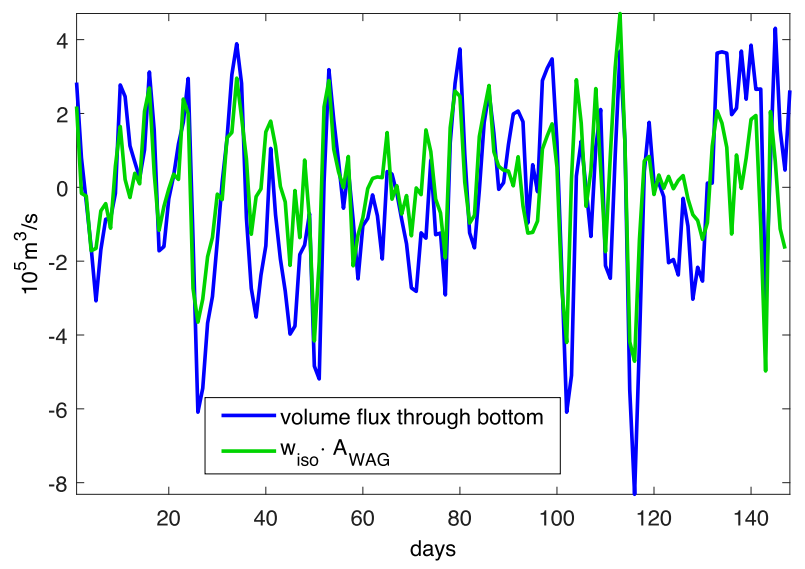

FIG. 8. Eulerian WAG net vertical volume transport in blue and mean velocity of the $\sigma_{\theta}=27.5 \mathrm{~kg} \mathrm{~m}^{-3}$ isopycnal multiplied by the area of the WAG in green.

This is consistent with the high-frequency fluctuations in advective fluxes in our results. However, as the cumulative advective fluxes show, additions of lower-salinity water are overwhelmed by the additions of higher-salinity water. Our interpretation points toward the formation of the salinity minimum during the reformation of the gyre. An example of the salinity patterns at 50-m depth (within the minimum) in the reforming WAG is shown in Figs. 10e and 10f, demonstrating the plausibility of the creation of the salinity minimum. The newly formed WAG in our model is warmer and fresher than water to the north or east because it is fed during the formation process by a warm and fresh coastal AJ.

To summarize, the leading-order terms in our Eulerian heat and salt budgets are advection and changes in heat and salt storage in time, followed by surface forcing and vertical diffusion. Over the 148 model days analyzed, the gyre is getting cooler and saltier on average so that both temperature and salinity extrema in the WAG are reduced. It is important to note that while the changes in salinity are nearly monotonic, changes in heat are not. While these Eulerian analyses of a fixed control volume may not exactly correspond to the dynamic WAG, we have observed no large variations in and no sustained migration of the WAG position over the period under consideration. This suggests that our conclusions from the Eulerian budgets might also hold for a time-evolving Lagrangian WAG. We next examine a vorticity budget to confirm whether all Eulerian budgets show advection driving a mean erosion of the gyre.

\section{b. Vorticity and circulation}

The WAG has anticyclonic circulation (see Fig. 4) and past work has examined its formation (Whitehead and Miller 1979; Preller 1986; Bormans and Garrett 1989) and variability (Heburn and La Violette 1990; Renault et al. 2012; Peliz et al. 2013). In a study on the collapse of the gyre, Sánchez-Garrido et al. (2013) found that the flux of cyclonic vorticity through the Strait of Gibraltar and into the Alboran Sea can have a destabilizing effect on the WAG. However, a vorticity budget for 

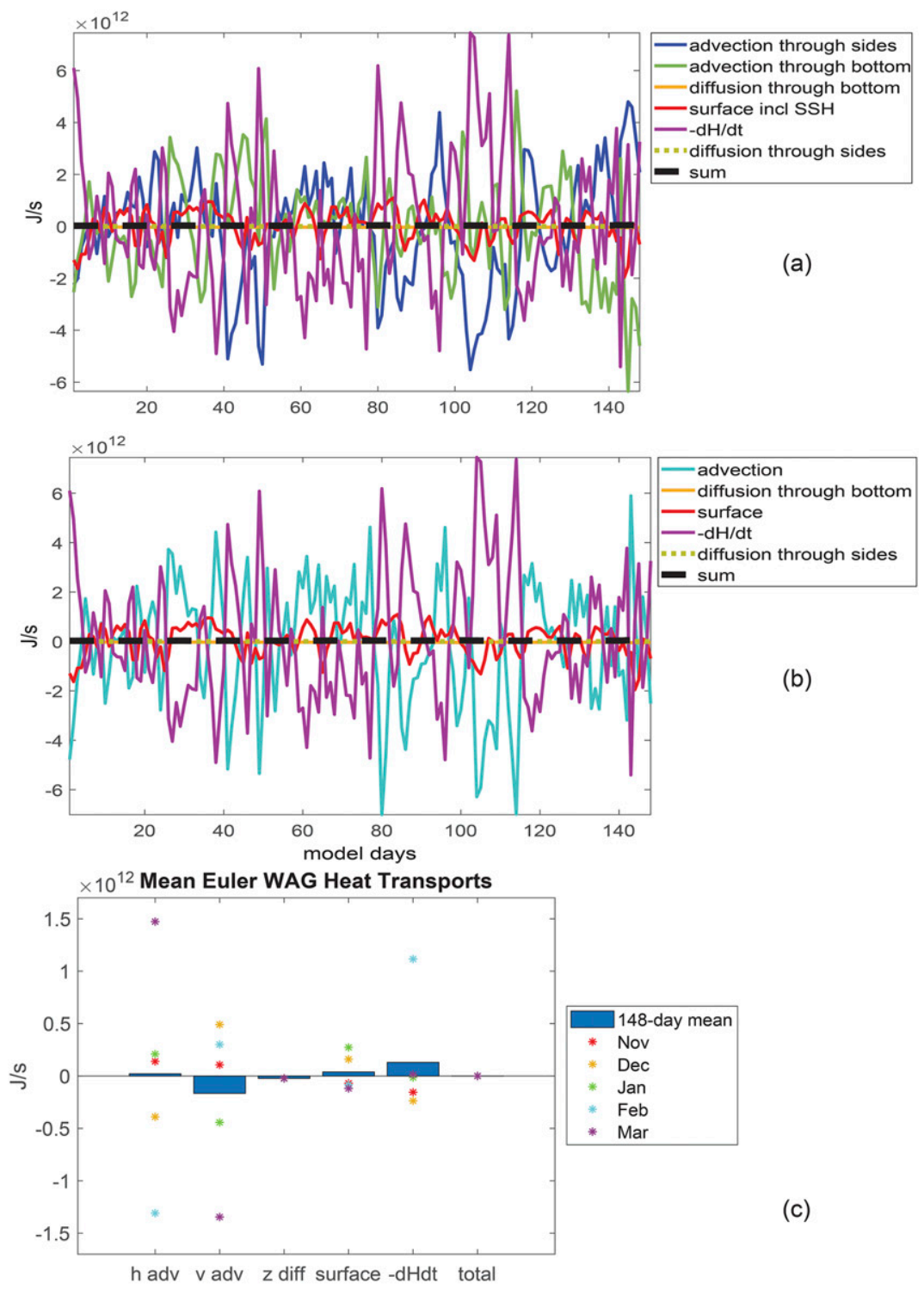

(c)

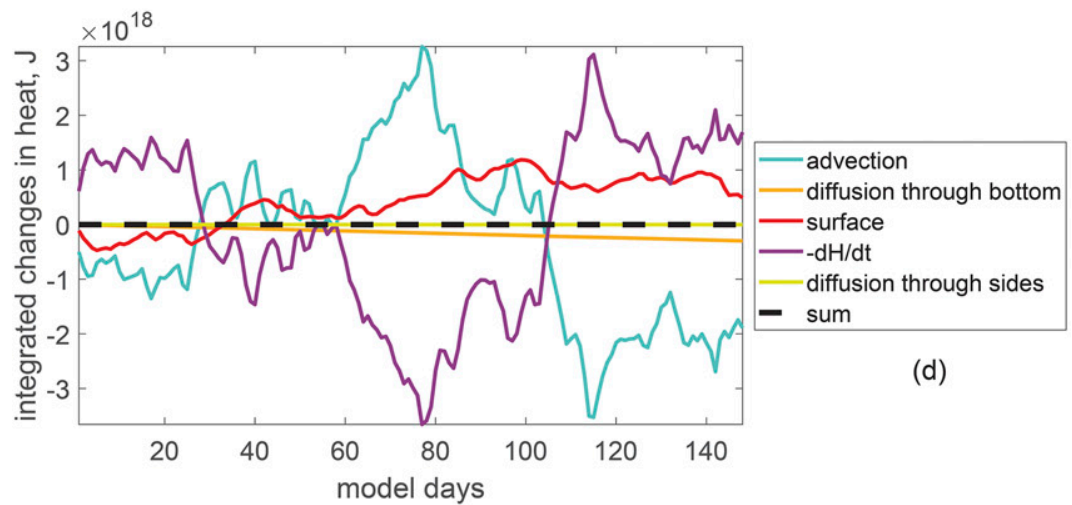

FIG. 9. (a) Eulerian WAG heat budget $\left(\mathrm{J} \mathrm{s}^{-1}\right)$, with the transports of heat by the volume transports of the mean gyre temperature, $16.99^{\circ} \mathrm{C}$, removed. (b) As in (a), but advection combined. (c) Time mean of heat fluxes $\left(\mathrm{J} \mathrm{s}^{-1}\right)$ from (a), over the full period (bars) and each month (stars). Terms are, from left to right, advection through the sides, advection through the bottom, vertical diffusion, surface forcing, $-H(t)+H(0)$, and the sum of the terms. (d) Cumulative heat fluxes (J) from (b). 


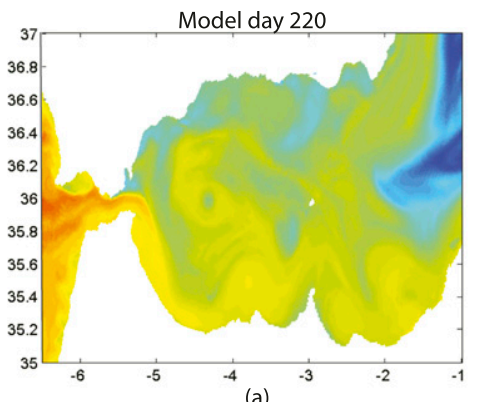

(a)

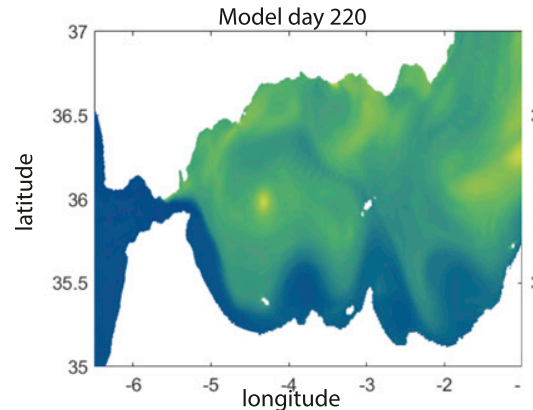

(d)

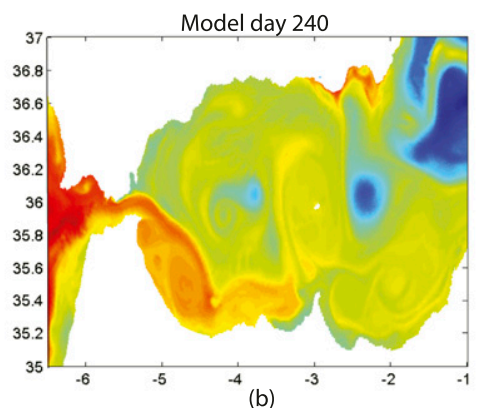

(b)

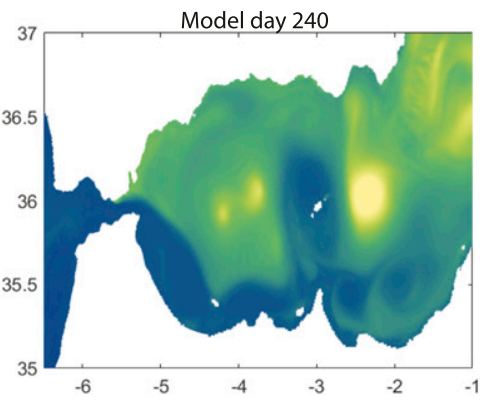

(e)
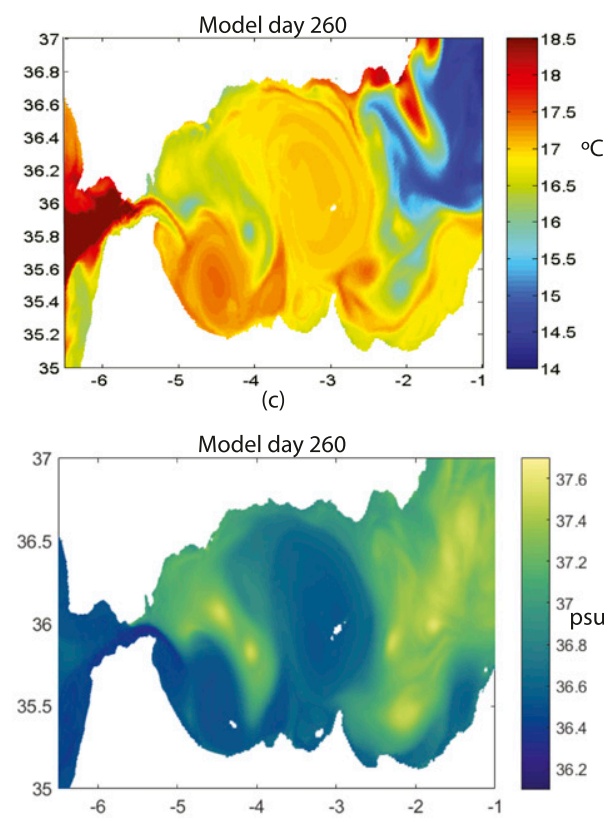

(f)

FIG. 10. (a)-(c) Potential temperature at $25 \mathrm{~m}$ and (d)-(f) salinity at $50 \mathrm{~m}$ on simulation days 220,240 , and 260 . The depth of $50 \mathrm{~m}$ is within the depths where the salinity minimum is apparent in our analyzed period and in observations; $25 \mathrm{~m}$ is above this, where temperature and salinity vary similarly. Day 220 is typical of the coastal circulation mode where the AJ is attached to the African coast, similar to observations in June 1986 (Heburn and La Violette 1990) and February 1998 (Vargas-Yáñez et al. 2002). The remaining days show how the AJ carries Atlantic water that reforms the WAG.

the WAG itself was not presented. Here we examine the vorticity budget for the Eulerian WAG to understand whether, similarly to the mean deterioration of the temperature maximum and salinity minimum, advection is causing a gradual spindown of the WAG.

A simple measure of the spin or rotation of the gyre at a given depth $z$ is the circulation $\Gamma=\oint_{C_{n}} \mathbf{u}_{h} \cdot d \mathbf{s}$ about a contour $C_{n}$ that is defined by the intersection of the constant-depth plane with the edge of the control volume we have been using. The circulation is also the integral of the vertical component $\zeta$ of relative vorticity over the horizontal area $A_{n}$ enclosed by $C_{n}$. Then the rate of change of the circulation is

$$
\frac{d}{d t} \iint \zeta d A_{n}=\frac{d}{d t} \iint \nabla \times \mathbf{u}_{h} \cdot d \mathbf{A}=\frac{d}{d t} \oint_{C_{n}} \mathbf{u}_{h} \cdot d \mathbf{s}=\frac{d \Gamma}{d t} .
$$

An equation for the rate of change of $\Gamma$ can be obtained by integrating the tangential components of the momentum equation about $C_{n}$, or by integrating the vertical vorticity equation over $A_{n}$. In either case the result is

$$
\begin{aligned}
\frac{d \Gamma}{d t} & +\oint_{C_{n}} \zeta_{a} \mathbf{u}_{h} \cdot \hat{\mathbf{n}} d \mathbf{s}-\oint_{C_{n}} w \zeta_{H} \cdot \hat{\mathbf{n}} d \mathbf{s} \\
& =\oint_{C_{n}} \frac{-1}{\rho} \nabla P \cdot d \mathbf{s}+\oint_{C_{n}} \nu \nabla \zeta \cdot \hat{\mathbf{n}} d \mathbf{s}+\oint_{C_{n}} \mathbf{F} \cdot d \mathbf{s},
\end{aligned}
$$

where $d \mathbf{s}$ is a differential length vector along $C_{n}, \hat{\mathbf{n}}$ is the outward unit vector perpendicular to $C_{n}, \zeta_{a}$ is the vertical component of absolute vorticity, $\zeta_{a}=\zeta+f, \zeta_{H}$ is the horizontal component of the relative vorticity, $P$ is pressure, $\nu$ is viscosity determined by KPP parameterization (Large et al. 1994), and $\mathbf{F}$ is a body force representation of wind stress and drag. Equation (4) states that changes in circulation about a given contour $C_{n}$ are due to the horizontal advection of the vertical component of absolute vorticity across $C_{n}$, the vertical advection of the horizontal component of vorticity across $C_{n}$, the effect of baroclinicity, the viscous diffusion (both vertical and horizontal), and body forcing acting about $C_{n}$, comprised of wind stress at the surface and drag in grid cells adjacent to the bottom. This budget gives the balance of terms for the horizontal area of the gyre at any $z$. To compare with our previous results for salt, heat and volume, we form a sum of area vorticity budgets at different $z$, weighted by the thickness of the model layer, to get a volume-integrated budget for the Eulerian WAG; details of computation from the MITgcm diagnostic outputs are in the appendix.

The resulting volume-integrated circulation budget has changes in circulation with time, total advection (horizontal and vertical advection of absolute vorticity), and horizontal diffusion as its largest daily terms, followed by drag, vertical diffusion, and wind stress (Fig. 12a); these six processes dominate the vorticity budget. Wind stress is shown combined with vertical diffusion because there is no vertical diffusion of momentum across the air-sea interface but instead a stressinduced transfer from the wind; thus the vertically integrated combination of diffusion and wind stress is the wind stress at the air-sea interface minus the diffusive flux at the bottom of the gyre. The total advection term includes the advection of planetary vorticity across $C_{n}$, equivalent to the vortex stretching 

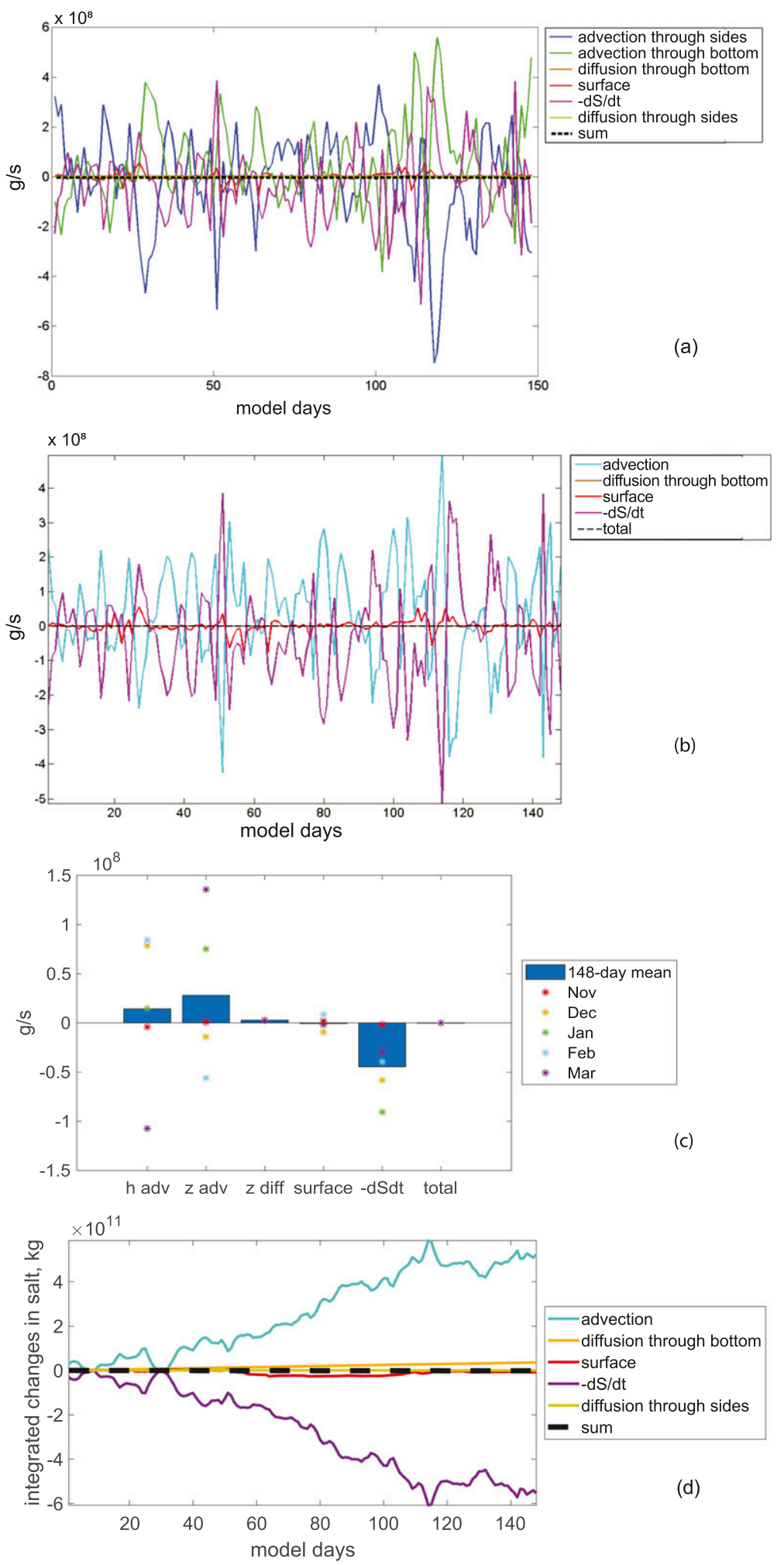

FIG. 11. (a) Eulerian WAG salt budget $\left(\mathrm{g} \mathrm{s}^{-1}\right)$, with the transports of salt by the volume transports of the mean gyre salinity, 36.61, removed. (b) As in (a), but advection combined. (c) Time-mean of fluxes $\left(\mathrm{g} \mathrm{s}^{-1}\right)$ in (a), over the full period (bars) and each month (stars). Terms are, from left to right, advection through the sides, advection through the bottom, vertical diffusion, surface forcing, $-S(t)+$ $S(0)$, and the sum of the terms. (d) Cumulative fluxes (kg) from (b). 
term, which has daily values of similar magnitude to the advection of relative vorticity $\left( \pm 20 \mathrm{~m}^{3} \mathrm{~s}^{-2}\right.$, see the online supplemental material for details) consistent with the heaving of the $\sigma_{\theta}=27.5 \mathrm{~kg} \mathrm{~m}^{-3}$ surface discussed with the volume budget. The baroclinic pressure contribution is five orders of magnitude smaller than the smallest of the six main terms, so we will not discuss it further. Without the baroclinic pressure term, the sum (error) of the remaining budget is still less than $1.5 \%$ of the smallest remaining term. Daily values of these terms are highly variable, with all but drag taking both signs, and there are no clear correlations between them.

The volume-integrated means of the six dominant terms (Fig. 12b) show that horizontal and vertical diffusion of vorticity serve to maintain the Eulerian WAG's anticyclonic rotation while advection of vorticity (fluxes due to resolved eddies), wind stress, and drag slow it down, giving a positive mean change in circulation. However, the 148-day mean increase in circulation, indicating a slight loss of anticyclonic rotation, does not represent a large or monotonic change. While the daily time series show rapid changes in the values of all terms and signs of most, monthly means of the dominant circulation budget terms (color dots in Fig. 12b) demonstrate that for total advection, diffusion, and drag the averaging period does not change their signs. Only wind stress and the changes in circulation in time take both signs in the monthly means, and there is no trend in their monthly-mean values. The cumulative fluxes of the circulation budget terms (Fig. 12c) show that the drag, total advection, and horizontal diffusion are nearly monotonic, indicating that their effects on the WAG's rotation are consistent; these are the largest terms in the mean. While the slowing effects of drag are expected, total advection is also decreasing the rotation in this region. The advection of planetary vorticity is a portion of this contribution, $0.71 \mathrm{~m}^{3} \mathrm{~s}^{-2}$ in the mean (green dash-dot line in Fig. 12c), related to a shallowing of the $\sigma_{\theta}=27.5 \mathrm{~kg} \mathrm{~m}^{-3}$ surface by $14 \mathrm{~m}$ over the analyzed period, but the advection of relative vorticity $\left(4.89 \mathrm{~m}^{3} \mathrm{~s}^{-2}\right.$ in the mean, blue dash-dot line in Fig. 12c) contributes more to the slowing of the gyre. The heaving of the $\sigma_{\theta}=27.5 \mathrm{~kg} \mathrm{~m}^{-3}$ surface (shown previously in Fig. 8) partially drives the planetary vorticity advection, $r=0.51$, with a good match of many of the largest daily values, through a stretchingsquashing effect. The relative vorticity advection adding cyclonic vorticity to the Eulerian WAG is consistent with conclusions from Sánchez-Garrido et al.'s (2013) work, which showed that advection of cyclonic relative vorticity into the WAG was large shortly before the gyre's collapse.

The depth dependency of these terms (Fig. 12d) is generally consistent with the depth-integrated means. At all depths the anticyclonic circulation is slowly decaying (positive $d \Gamma / d t$ ) due in part to drag, opposed by horizontal diffusion. There are some differences at the surface: here, advection supports the anticyclonic circulation and the difference between the wind stress and the downward diffusion of vorticity opposes it. Below $50 \mathrm{~m}$, the vertical diffusion is near zero but takes both signs.

The continued rotation of the Eulerian WAG is supported by diffusion of anticyclonic vorticity into the gyre, whereas advection of vorticity, drag, and wind stress lead to decay.
Contributions of horizontal diffusion to the driving of the gyre have not been previously discussed, to our knowledge. However, it can be thought of as the cumulative effect of lateral stress due to unresolved eddy fluxes: where flow outside the gyre is faster than that inside in the same direction, diffusion will move momentum of that direction into the slower gyre. Near the surface, the AJ is faster than the WAG parallel to their boundary (Fig. 4) and diffusion of momentum can thus support the anticyclonic rotation. At depth, the WAG separates from the Moroccan coast with a portion of the outflowing deep water between them (velocities in Fig. 13). At these depths, the water outside the control volume is moving faster than that inside at many locations, including this outflow, and can support the anticyclonic rotation through the lateral diffusion of momentum. A contribution from the deep outflow to the anticyclonic vorticity of the WAG is consistent with Bryden and Stommel (1982).

To summarize, the vorticity budget for the Eulerian WAG shows that by the end of our 148-day analysis period, the gyre's rotation has slowed down slightly. Its continued anticyclonic rotation is supported by the wind stress and diffusion of vorticity. Advection and drag add cyclonic vorticity to the Eulerian WAG. The large role of advection in the vorticity budget is consistent with its large role in the salt and heat budgets.

\section{Lagrangian analysis}

From the results of the Eulerian analysis of the WAG, there appears to be a large transport of water across the stationary northern boundary and bottom. The fluctuating volume transport across the fixed bottom of the Eulerian WAG is associated with the heaving of the $\sigma_{\theta}=27.5 \mathrm{~kg} \mathrm{~m}^{-3}$ surface. Since little transport is expected to occur across this surface, it is reasonable to consider a Lagrangian definition of the WAG in which the instantaneous $\sigma_{\theta}=27.5 \mathrm{~kg} \mathrm{~m}^{-3}$ surface is chosen as the bottom and a time-dependent lateral boundary is constructed from evolving material surfaces. An intuitive and convenient choice of an instantaneous lateral boundary of the Lagrangian WAG consists of segments of stable and unstable manifolds connecting hyperbolic regions near Ceuta and Melilla. Exchange of water across this time-evolving boundary can then be conveniently described through a turnstile lobe mechanism. The reader is referred to Samelson and Wiggins (2006) for varied textbook examples of the methodology. Miller et al. (2002) describes an example that is particularly relevant to the case at hand.

The basic approach can be illustrated by first imagining that the near-surface WAG is steady, incompressible, and 2D (Fig. 14a), and bounded by a streamline that extends between hyperbolic stagnation points $H_{1}$ and $H_{2}$. The former corresponds to Ceuta, where the AJ separates from the Moroccan coastline, while the latter lies at the tip of a small peninsula near Melilla, where flow splits into eastward and westward branches. If time dependence is now introduced (Fig. 14b) it is generally the case that the hyperbolic regions $H_{1}$ and $H_{2}$ are preserved, not as stagnation points, but as possibly moving points on the boundary about which the same hyperbolic behavior occurs, i.e., the fluid still flows toward the hyperbolic region in the alongshore direction and away from the 

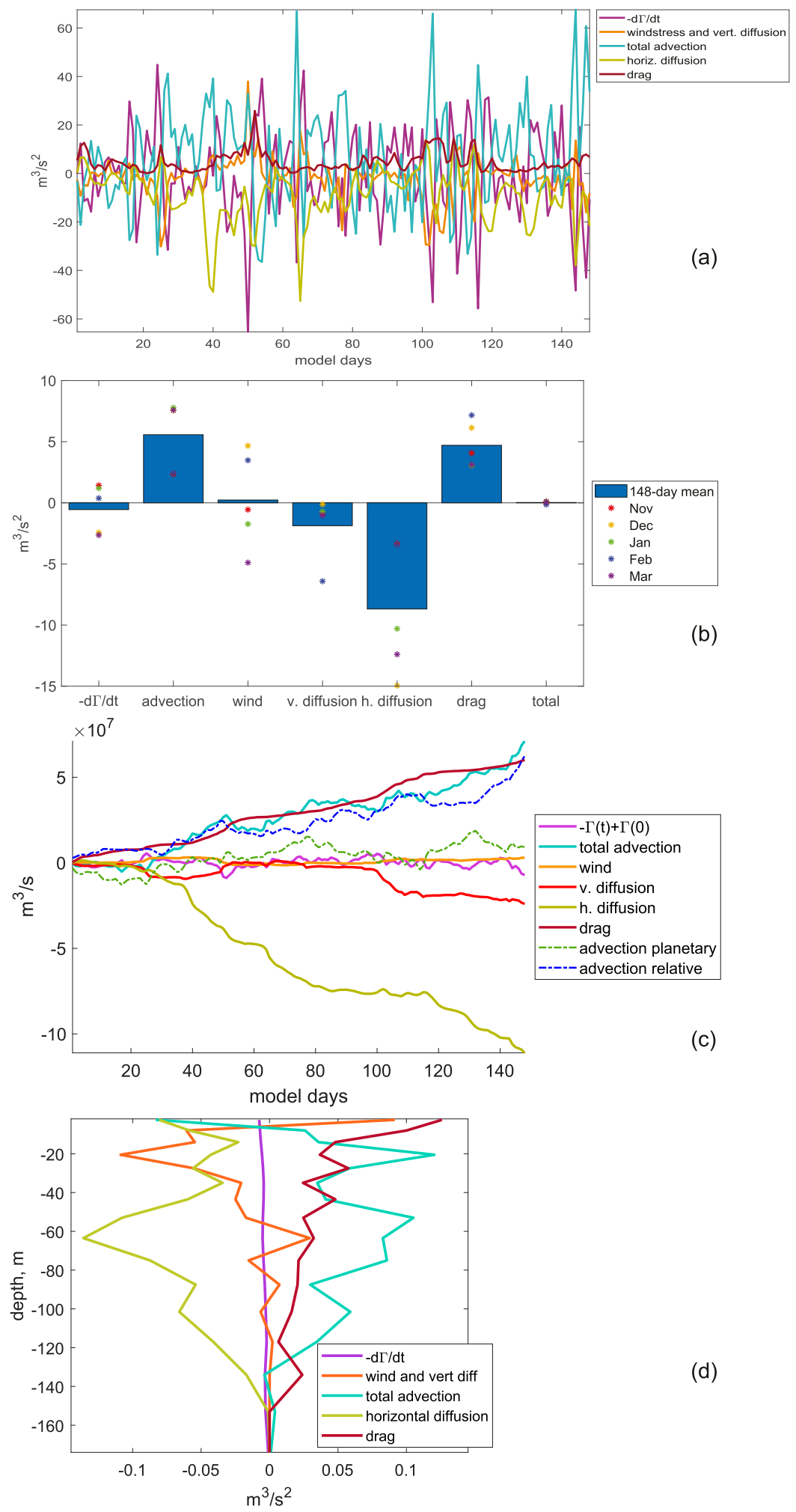

(d)

FIG. 12. (a) Daily volume-integrated vorticity budget terms $\left(\mathrm{m}^{3} \mathrm{~s}^{-2}\right)$. (b) Time means of the terms, 148-day period in blue bars, colored stars for each month's means. (c) Cumulative fluxes $\left(\mathrm{m}^{3} \mathrm{~s}^{-1}\right)$, with components of total advection, the advection of planetary vorticity, and advection of relative vorticity, shown as dash-dot. (d) Depth dependence of the time-mean terms $\left(\mathrm{m}^{2} \mathrm{~s}^{-2}\right)$. 


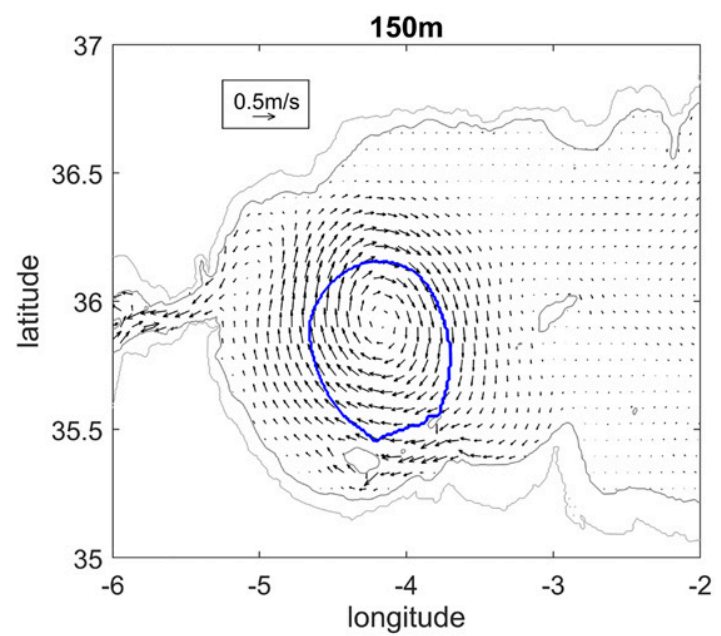

(a)

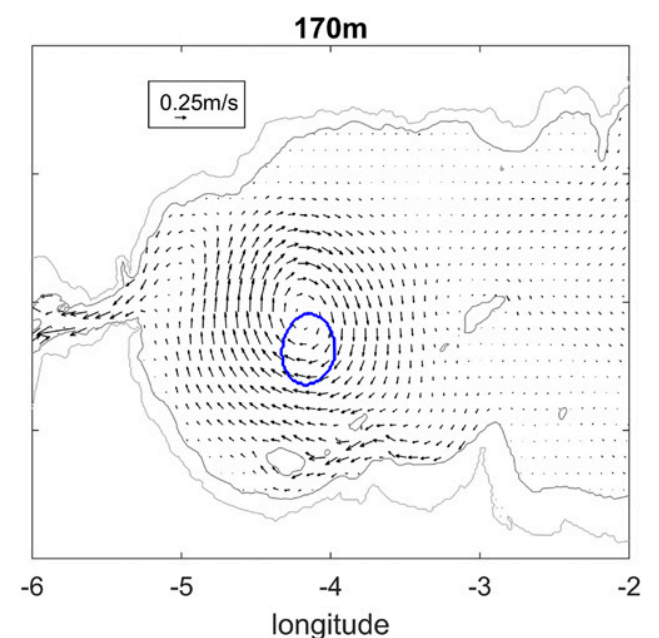

(b)

FIG. 13. Mean velocity at (a) 150- and (b) 170-m depths over the 5 months analyzed; light gray contour is the coast, dark gray contours are the 150- and 170-m isobaths in (a) and (b), respectively, and blue is the edge of the Eulerian WAG at these depths. Note the westward current along the southern coast that connects to the outflow through the Strait of Gibraltar.

hyperbolic region in the cross-shore direction for the western hyperbolic region and vice versa for the eastern. We may define the stable manifold of $\mathrm{H}_{2}$ as the contour consisting of material that approaches $H_{2}$ asymptotically in time. Similarly, the unstable manifold of $H_{1}$ consists of material that approaches $H_{1}$ asymptotically in backward time (departs from it in forward time). In the steady, incompressible, 2D flow, these objects are the same and form the separating streamline that defines the WAG boundary. In the unsteady case they are generally not the same and intersect each other in the manner shown in Fig. 14b.

An advantageous choice of a boundary for an evolving gyre consists of a combination of a segment of the stable manifold emanating from $\mathrm{H}_{2}$ and a segment of the unstable manifold emanating from $H_{1}$, the two joined at the intersection point labeled $I_{n}$. An example is represented by the dashed curve in Fig. $14 \mathrm{~b}$, and it is clear that this new boundary connects $H_{1}$ to $H_{2}$. As the flow evolves, the intersection point $I_{n}$ drifts east (Fig. 14c); after some time, such as when $I_{n}$ passes a given longitude, a new manifold intersection point, former $I_{n-2}$, becomes the boundary-defining intersection and the boundary is then instantaneously redefined. As a result of this redefinition, the fluid in lobe $L_{n}$ in Figs. $14 \mathrm{~b}$ and $14 \mathrm{c}$ is carried out of the gyre, whereas the fluid in lobe $L_{n-1}$ is carried in. This accounts for the term "turnstile lobes," which implies an exchange of fluid. When the gyre boundary is defined this way, lobes $L_{n}$, $L_{n-2}$, etc. contain fluid that is destined to leave the gyre, whereas lobes $L_{n-1}, L_{n-3}$, etc., contain fluid that is destined to enter the gyre. Because the lobes experience rapid stretching and folding as they move toward $H_{2}$, the region near the gyre's periphery, which is occupied by the lobes, is characterized by more vigorous stirring than the gyre's core region, where manifolds, and thus lobes, do not penetrate. Consistent with this idea, Sayol et al. (2013) estimated finite-size Lyapunov exponents at the sea surface from surface velocities of the Western Mediterranean Operational Model, and showed that the region around the boundary of the Alboran Gyre is, by this measure, one of enhanced stirring (although the authors did not compute manifolds or identify turnstile lobes).

\section{a. Manifold construction}

To construct the 3D unstable manifold that begins near the Ceuta separation point, we begin at the sea surface and employ a "direct" manifold estimation method that consists of seeding ensembles of fluid parcels surrounding the vicinity of this point (Miller et al. 2002; Mancho et al. 2003; Samelson and Wiggins 2006; Rypina et al. 2010; Branicki and Kirwan 2010). We do not know the exact location of the hyperbolic trajectory $H_{1}$, except for the important fact that it always exists near Ceuta. Because of the hyperbolic nature of motion in this region, the seeded parcels will tend to flow toward $H_{1}$ in the alongshore stable direction, and will depart from it in the offshore unstable direction. The seeding of parcels near Ceuta and tracking of their trajectories using horizontal surface velocities will thus result in a material contour that approximates a segment of the unstable manifold at the surface. Repeating this calculation by seeding groups of parcels at different depths and tracking their path along the corresponding isopycnals results in material contours that lie along the subsurface expression of the unstable manifold on that isopycnal. The manifold segments on different isopycnals can then be stacked together vertically to produce a $3 \mathrm{D}$ picture. In doing so, we assume that all subsurface motion lies along potential density surfaces, and we use 6 surfaces in our calculation: the sea surface and $\sigma_{\theta} \in\{26.3,26.5$, $26.75,27,27.5\} \mathrm{kg} \mathrm{m}^{-3}$. The choice to use isopycnal surfaces is in part practical, as it reduces the computationally intensive estimation of large numbers of 3D trajectories to the computation of quasi-2D trajectories along isopycnal surfaces. We tested this 


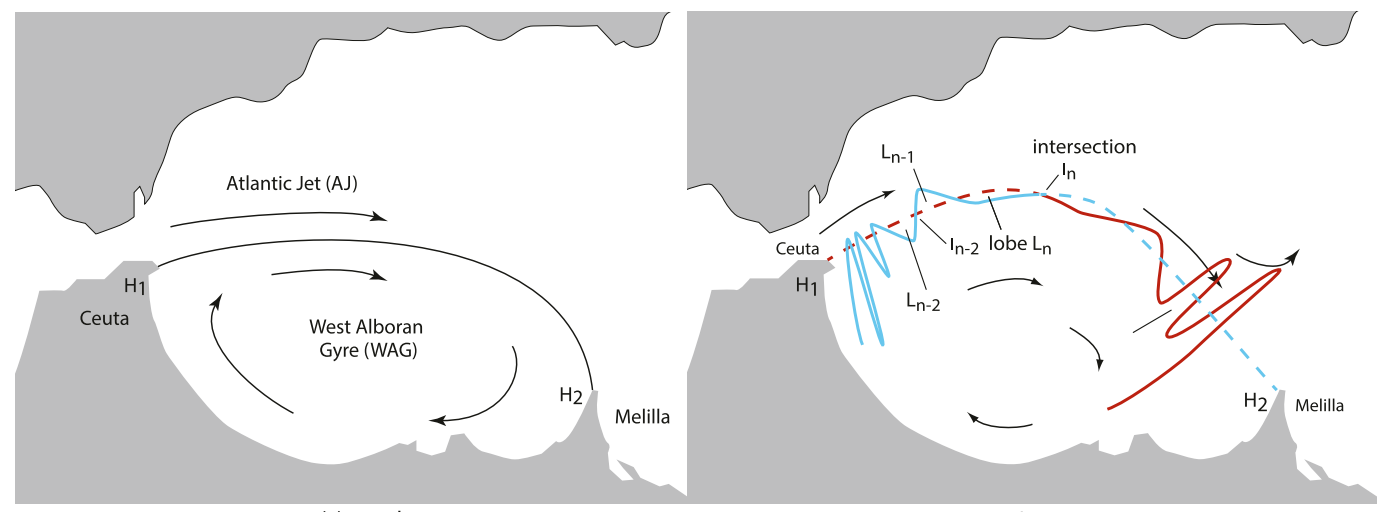

(a) steady

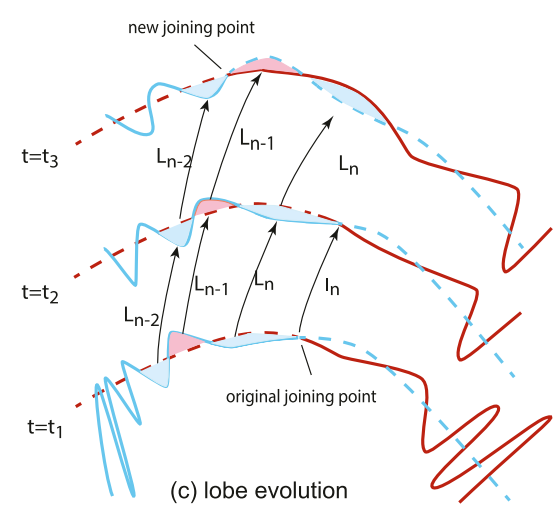

(b) time-varying

(c) lobe evolution

FIG. 14. Lagrangian geometry. (a) Steady case. Water is trapped in the gyre, south of the material contour (black curve) connecting hyperbolic points $H_{1}$ and $H_{2}$, for all time. (b) Periodic case. Unstable (red) and stable (blue) manifolds. Lobe $L_{n-2}$, between manifold intersections $I_{n-2}$ and $I_{n-3}$, maps to $L_{n}$ after one period and will transport water out of the WAG, whose piecewise boundary is the dashed curve. Lobe $L_{n-1}$ transports water into the WAG. (c) Enlargement of the northern portion of the gyre showing the movement of individual lobes in the presence of more general time dependence. The formal boundary of the gyre still consists of the union of the dashed curves, which join at the moving intersection point $I_{n}$ during the time interval $t_{1} \leq t<t_{3}$. At $t=t_{3}$ the joining point is redefined as shown, instantaneously altering the boundary and placing lobe $L_{n}$ outside of, and lobe $L_{n-1}$ inside of, the boundary.

assumption of along-isopycnal motion for a set of 1000 trajectories initialized on the $\sigma_{\theta}=27 \mathrm{~kg} \mathrm{~m}^{-3}$ surface on model day one and integrated for 14 days using both the full three-dimensional velocity field and the along-isopycnal assumption. We found that horizontal separations were $3.7 \mathrm{~km}$ on average, with $50 \%$ of separations within $1.4-5.3 \mathrm{~km}$ and $90 \%$ within $0.9-7.8 \mathrm{~km}$; these differences in position after 14 days are small compared to the trajectory's lengths, which were an average of $360 \mathrm{~km}$. In the vertical, the mean separation after 14 days was $5.6 \mathrm{~m}$ and $90 \%$ of separations were between 0.8 and $9 \mathrm{~m}$, again much smaller than the total vertical displacement of trajectories, which was $152 \mathrm{~m}$ on average. The surface corresponding to the stable manifold of $\mathrm{H}_{2}$ is calculated by seeding trajectories in the vicinity of Melilla and integrating backward in time.

Trajectories were seeded using 100 points distributed within a radius of $10 \mathrm{~km}$ of the topographic corners near Ceuta $\left(35.86^{\circ} \mathrm{N}, 5.35^{\circ} \mathrm{W}\right)$ and Melilla $\left(35.4^{\circ} \mathrm{N}, 3.05^{\circ} \mathrm{W}\right)$. For the trajectory integration, we use an explicit Runge-Kutta 4.5-order scheme with linear interpolation in space and time from dailyaveraged horizontal velocities on a given isopycnal. The integration time is 8 or 14 days, with daily reseeding along the curve of the manifold to have a spacing of $2 \mathrm{~km}$ or less, allowing the shape of the manifold segment to be well resolved. The 8-day integration period is slightly longer than the typical transit time from Ceuta to Melilla of about 5 days at the surface; the 14-day integration is slightly longer than the typical transit time of about 11 days along the $\sigma_{\theta}=27.5 \mathrm{~kg} \mathrm{~m}^{-3}$ isopycnal. Similar methods have been used by, e.g., Miller et al. (2002), Mancho et al. (2003), and Rypina et al. (2010). It is important to note that this direct manifold estimation only works with isolated hyperbolic trajectories, when there are no other manifolds in the vicinity, and when the hyperbolic trajectory is surrounded by the initial seeding. Also, the resulting contour is only an approximation of a segment of the true manifold. Despite these caveats, the direct manifolds calculated this way generally have a good correspondence with ridges of finite-time Lyapunov exponent fields, which are often used as (somewhat noisy) proxies for finding manifolds (Branicki and Kirwan 2010; Rypina et al. 2010).

\section{b. Stirring region and core}

Manifolds can be used to delineate the WAG from its surroundings in the manner schematically shown in Fig. 14b. The exchange of water across this boundary occurs through turnstile 

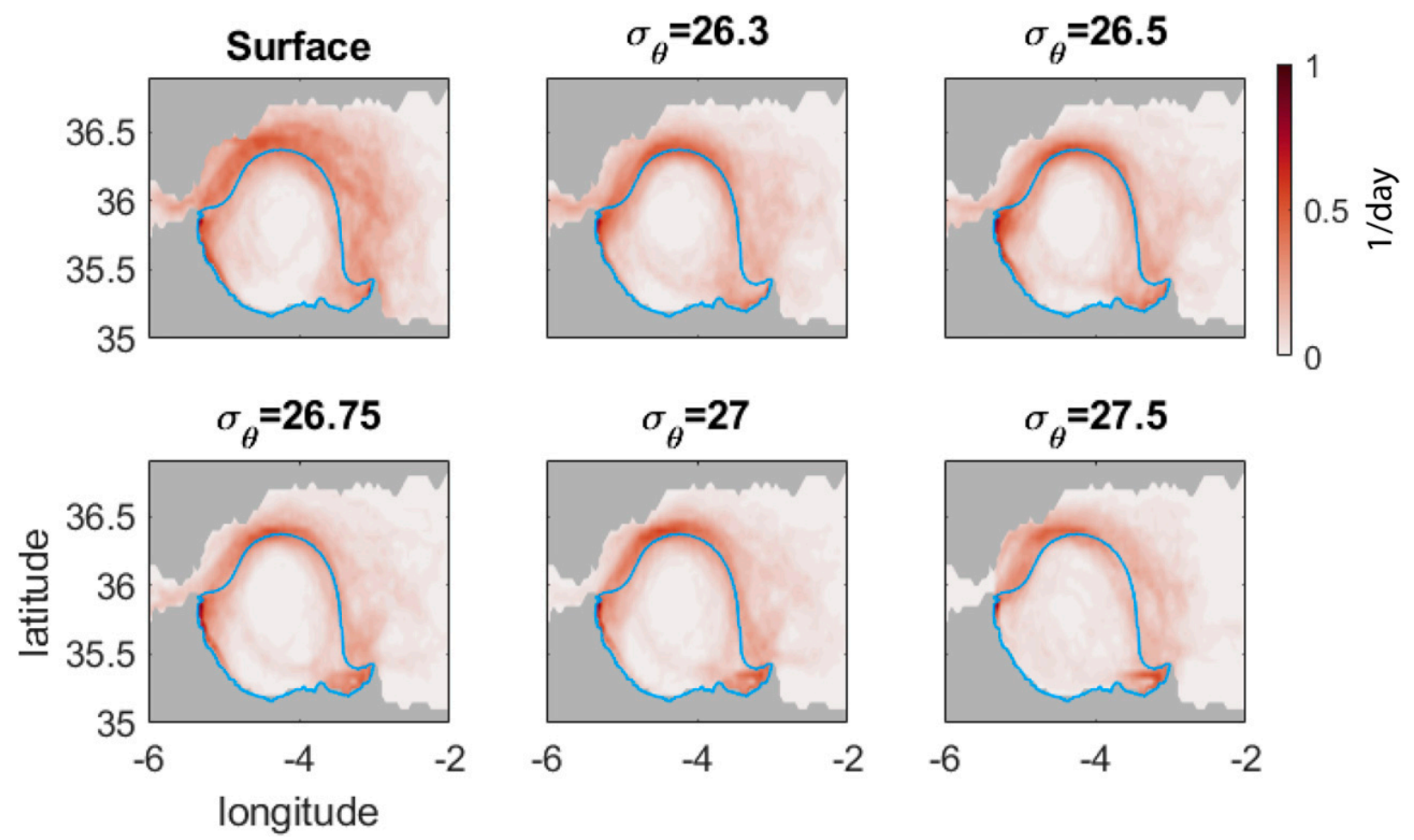

FIG. 15. Frequency at which a manifold segment crosses each location, reds. Manifolds computed with 2-km resolution from daily horizontal velocities over an 8-day integration. Blue curves are the Eulerian WAG horizontal boundary.

lobes and therefore is also defined by the manifolds. Thus, we will define the finite-time stirring or exchange region as the region where our calculated manifold segments are present, and we will identify the geographical extent of this region using spatial frequency maps.

In our analysis, we have computed manifold segments over 8 and 14 days on six surfaces. The locations of these manifold segments during 120 days of analysis (model days 15-134, using velocities from the 148 days analyzed in the Eulerian section) are used to create a map of their frequency of visiting small subregions, $0.05^{\circ}$ squares, of the Western Alboran. The frequency maps on each of the six surfaces is shown in Fig. 15 for 8-day manifolds. Regions with a high frequency of manifold occurrence are more likely to participate in the turnstile lobe mechanism on the time scale of the trajectory integration time, i.e., 8 or 14 days in our calculations. On all levels, the regions with high frequencies of manifolds overlap the area where the edge of the WAG is intuitively expected and generally align with the isohaline contour (blue contour in Fig. 15) that was chosen to represent the WAG boundary in our Eulerian study. The high probabilities along the coast in the interior of the gyre (west of Melilla and southeast of Ceuta) are due to variations in the position of the hyperbolic trajectory; southeast of Ceuta this is often related to small transient eddies. At the surface, the finite-time stirring region with high probability of manifold presence is also similar to that of high FSLEs in the Sayol et al. (2013) mean map. Interestingly, the nonzero manifold probabilities do reach the northwestern shore (although values diminish toward shore), suggesting that these coastal waters are, at times, involved in the turnstile lobe exchange with the WAG. In observations, this region has upwelling (Sarhan et al. 2000; Echevarría et al. 2009), which means this connection may be a source of nutrients for the ecosystems in the WAG. On deeper isopycnals, the region with high frequencies of manifold segments near the WAG perimeter is slightly narrower, with lower values at most locations. This decrease is due to slower velocities at depth yielding shorter manifold segments.

The complement of the finite-time stirring region at the periphery of the WAG is its finite-time core, a region where water is retained within the WAG over the corresponding integration times. We define the finite-time core as the region inside the WAG that is not crossed by any finite-time manifold segments (i.e., segments of manifold computed using an 8- or 14-day trajectory integration time) during our entire analysis period (i.e., 120 days) (Fig. 16); equivalently, this would be the region encircled by the zero-frequency contour in the frequency maps (Fig. 15). Fluid parcels that originate within this 8-day (or 14-day) finite-time core on any day during the 120 days of analyses will not participate in the turnstile lobe exchange mechanism over the subsequent 8 (or 14) days. Consistent with this interpretation, passive particles seeded inside the zero-frequency contours of the 14-day manifold frequency map stayed within the WAG over two weeks on all levels. However, this definition of the coherent core region is overly restrictive and differs from other Lagrangian coherent structure-based definitions, such as the least-stretching contour 


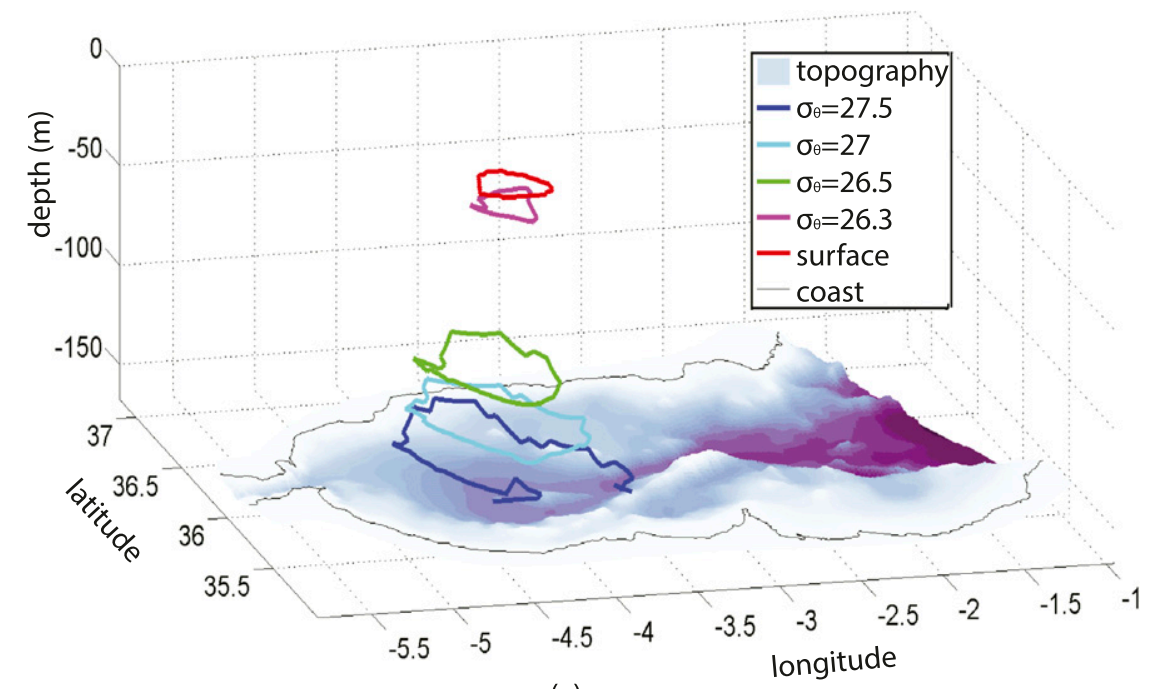

(a)

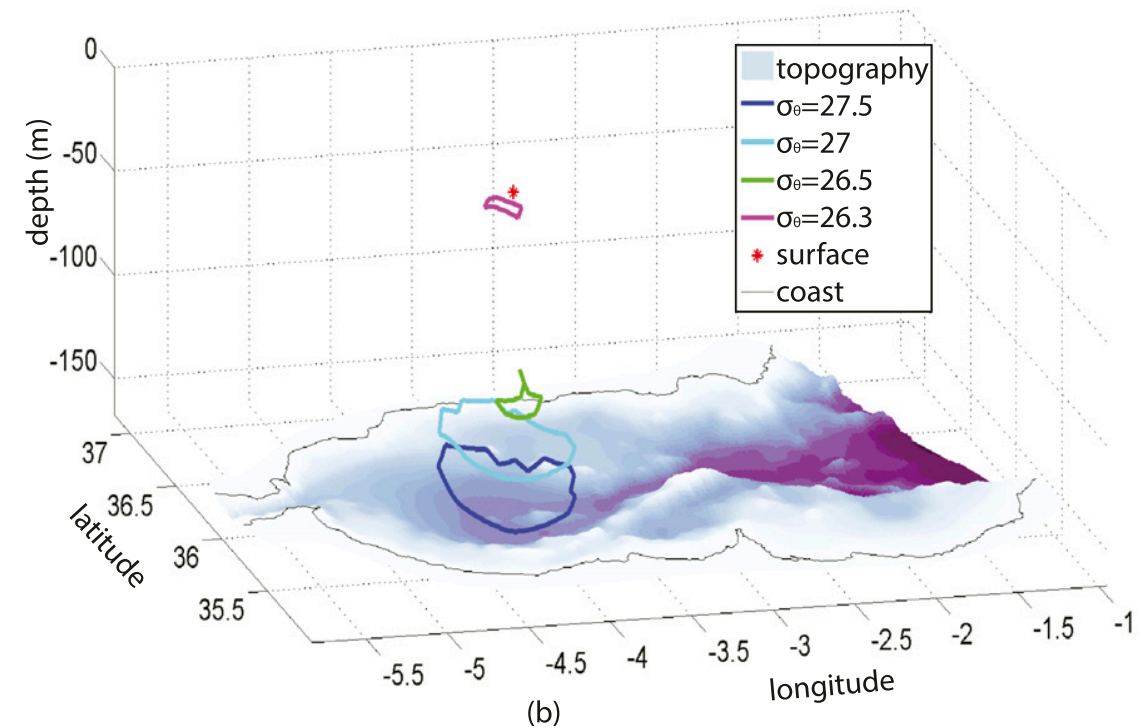

FIG. 16. The core region of the WAG. Zero contour of the manifold probability field from the surface and four isopycnals at their mean depths $\left(\sigma_{\theta}=26.75 \mathrm{~kg} \mathrm{~m}^{-3}\right.$ skipped for readability): (a) 8-day manifolds and (b) 14-day manifolds.

(Haller and Beron-Vera 2012), Lagrangian-average vorticity deviation (Haller et al. 2016), and diffusive transport barriers (Haller et al. 2018). The finite-time core region is larger on deeper isopycnals, again due to the same decreased velocities that thin the stirring region. In three dimensions, the finite-time core region is dome-like (Fig. 16).

The extent of the finite-time WAG core and stirring periphery depend on the manifold integration time: longer integration lengthens manifold segments, enlarges the finite-time exchange region that will participate in the turnstile lobe exchange over the chosen integration time, and decreases the size of the core. This is fully consistent with our intuition that larger regions of the WAG participate in the exchange with surroundings over longer integration time. The 14-day core region is smaller than the 8-day core, but shows the same structure with depth (Fig. 16). At the surface, the core has shrunk to a single point, indicating that all geographical areas within the western Alboran have participated in the turnstile lobe exchange at one time or another during the 120 days analyzed. However, the probability maps do not rule out the possibility of a Lagrangian core region that consists of material that moves around in the vicinity of the geographic center of the WAG and that does not participate in exchange. Indeed, maps of 8- and 14-day-long FTLEs on day 15 (Fig. 17) show a region with low stirring rates near the center of the WAG for both integration times. Particles seeded in that region on that day would stay close together and remain within the core over the next two weeks. However, particles seeded at the same location on another day might not because the low-stirring (low-FTLE) region evolves in time. Consistent with this interpretation, if we 

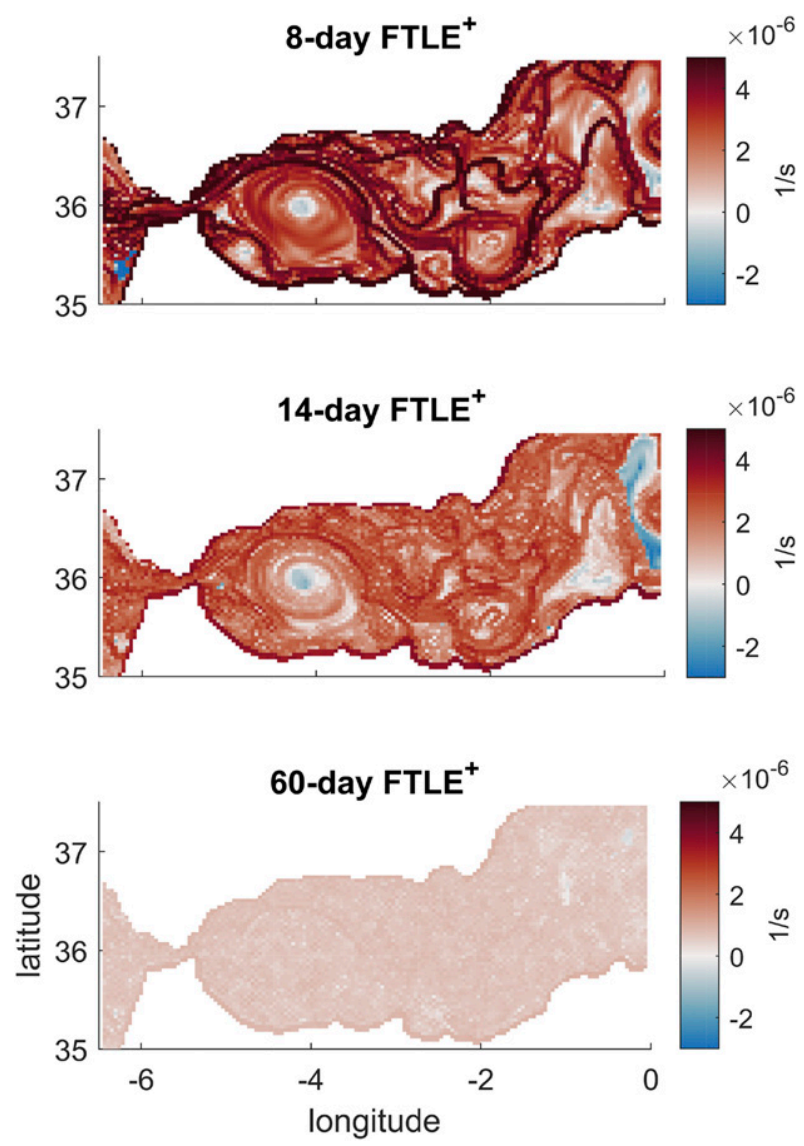

FIG. 17. Forward FTLE at the surface on model day 15 from several trajectory integration lengths.

recompute our manifold frequency maps for each month (instead of over 120 days), we see that the zero-frequency contours shift their positions slightly from month to month (example in the online supplemental material). Over a longer integration time of 30 or 60 days, there is no signal of a lowstirring region in the FTLE field (Fig. 17). This is likely related to the limited residence time $T_{\text {res }}$ within the western Alboran $-T_{\text {res }}$ sets the upper limit of the lifetime of a coherent core.

\section{c. Turnstile lobes and subduction}

As described above and illustrated in Fig. 14b, a Lagrangian WAG boundary can be constructed by "stitching together" a segment of the unstable manifold emanating from Ceuta and a segment of the stable manifold approaching Melilla. In the previous section, we described the stirring region where lobes and manifolds are located. Using those same manifolds at the surface and on five subsurface isopycnals, we can examine the shape of lobes in three dimensions.

The identification and tracking of lobes in complex flows is difficult given their propensity to become filamented, with their edges having many folds. Thus, identifying lobes is a nontrivial task, and connecting lobes between surfaces is a further challenge. We examined each day's manifolds on all depths in the first month analyzed in an effort to identify lobes. In that month, 33 lobes are found at the surface, 29 of which are also found on $\sigma_{\theta}=26.3 \mathrm{~kg} \mathrm{~m}^{-3}$, which also had 12 other lobes, not corresponding to the surface features; only 5 lobes were identified on deeper isopycnals that corresponded to the 29 on the first two surfaces. Given the considerable effort required to do the analysis on 5 isopycnals, we chose not to pursue the task of fully resolving the vertical structure of all the lobes as this would necessitate consideration of at least double the number of isopycnals. Closure of a 3D Lagrangian volume budget similar to the 2D budget presented in Miller et al. (2002) would require this additional degree of vertical resolution and possibly higher than daily temporal resolution; thus, we do not present such a budget, electing instead to present an example of one representative large lobe that we can resolve.

Figure 18 shows the evolution of a large lobe that extends from the ocean surface down through $\sigma_{\theta}=27 \mathrm{~kg} \mathrm{~m}^{-3}$. Although the lobe is clearly identifiable in the manifolds on the four upper surfaces for one week, it does not appear to be present below the $\sigma_{\theta}=27 \mathrm{~kg} \mathrm{~m}^{-3}$ isopycnal. The lobe is first identified outside and to the northwest of the WAG. As it moves clockwise, it crosses the WAG perimeter and enters the gyre. Then the lobe moves south toward the hyperbolic region near Melilla, shrinking in its horizontal extent and stretching vertically. This vertical stretching is due to subduction of its lower portions along isopycnal surfaces that slope down toward the center of the WAG.

This lobe is not unique in its behavior: it appears that lobes often subduct as they are entrained into the WAG. Lagrangian subduction and upwelling processes are a topic of extensive current research, and a complete characterization of the complex and multifaceted vertical exchange of trajectories is outside the scope of this work. However, we did a preliminary analysis to contextualize the lobe that we presented. To examine upwelling and subduction, we compute 21-day alongisopycnal trajectories from an initial grid across our model domain on the $\sigma_{\theta}=27.5 \mathrm{~kg} \mathrm{~m}^{-3}$ isopycnal; these trajectories are initialized on nine different model days spread evenly between days 1 and 126. Upwelling and subduction are defined as vertical displacements of at least $50 \mathrm{~m}$ over 8 days and net changes of at least $50 \mathrm{~m}$ over 21 days. We show the evolution of the position of those trajectories on $\sigma_{\theta}=27.5$ that subduct into the WAG region or upwell out of it (Fig. 19). Generally, trajectories that subduct begin to the north and east of the WAG and enter along a southwestward trajectory, similar to the presented lobe. There are also some subducting trajectories that begin in the Strait of Gibraltar. Trajectories that upwell from the WAG typically exit the gyre along a northeastward trajectory, moving into the eastern Alboran Sea. Overall, these trajectories are qualitatively consistent with the turnstile lobe exchange mechanism, connecting the horizontal and vertical exchange processes for this gyre. While this is a relatively small set of trajectories, the qualitative patterns hold across isopycnals $\left(\sigma_{\theta}=27 \mathrm{~kg} \mathrm{~m}^{-3}\right.$ and $28 \mathrm{~kg} \mathrm{~m}^{-3}$, not shown).

\section{Discussion}

We analyzed a numerical model of the Alboran Sea to understand the exchange of properties between the Western 


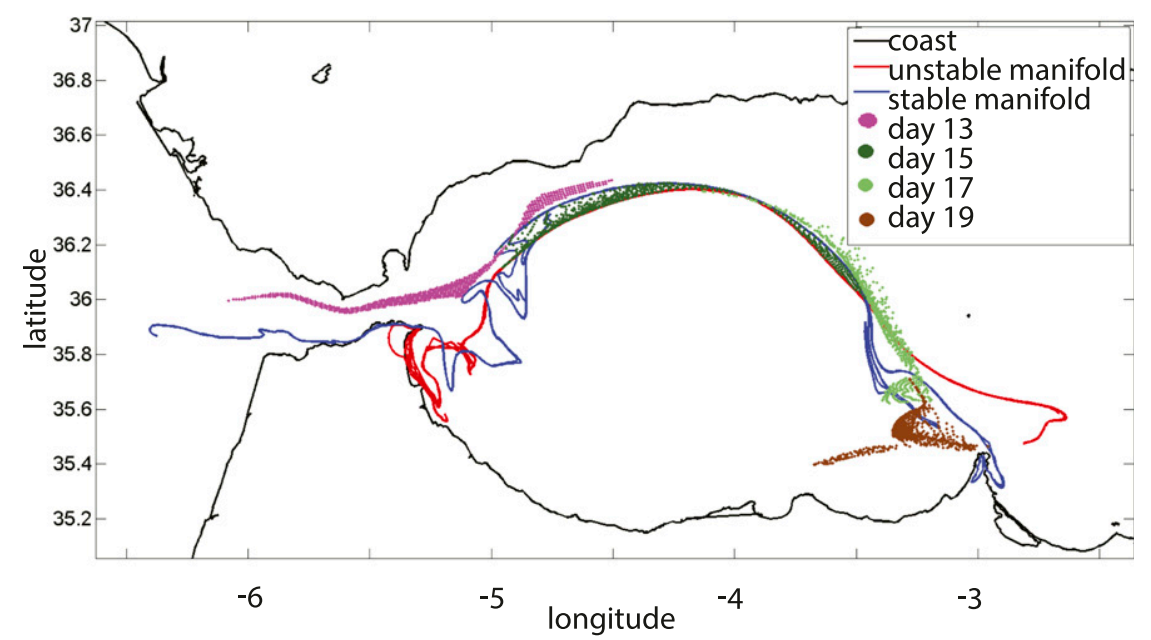

(a)

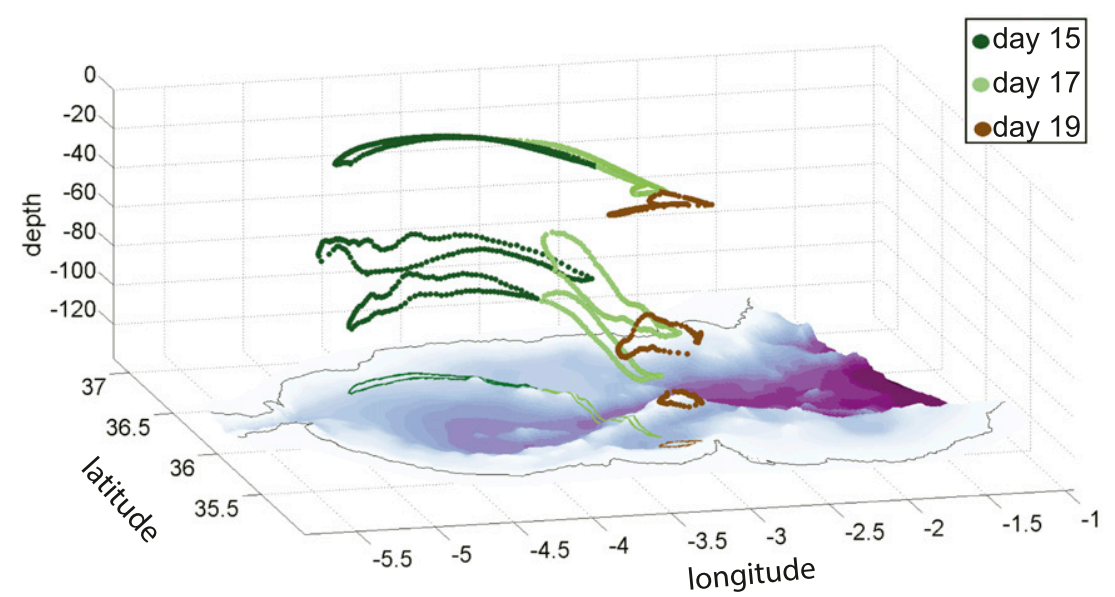

(b)

FIG. 18. Evolution of one lobe in 2D and 3D. (a) Points inside the lobe are shown on simulation days $13,15,17$, and 19 at the surface, with the manifolds from day 15 also shown. (b) Points show the edges of the lobe on days 15,17, and 19 at the surface and on isopycnals $\sigma_{\theta}$ $=26.5 \mathrm{~kg} \mathrm{~m}^{-3}$ and $\sigma_{\theta}=27 \mathrm{~kg} \mathrm{~m}^{-3}$, which are sections of the stable and unstable manifolds on those surfaces. Bottom topography is shown with purple deeper regions; green curves on the topography are the projection of the $\sigma_{\theta}=27 \mathrm{~kg} \mathrm{~m}^{-3}$ lobe edges on the three days.

Alboran Gyre and its surroundings using both Eulerian and Lagrangian methods. The model output had a realistic mean flow with a consistent WAG for about five months and our analyses aimed to elucidate the processes that drive changes in the water properties and dynamical characteristics of the WAG during the time when the WAG appears stable and does not show any signature of collapse.

From the Eulerian budgets for the WAG, we see a large erosion of the salinity minimum, temporally varying changes in the temperature maximum, and minimal changes in vorticity. The volume budget shows that the advective exchange of water across the fixed WAG edge, defined as the mean isohaline contour of 36.475 and mean $\sigma_{\theta}=27.5 \mathrm{~kg} \mathrm{~m}^{-3}$ surface, is large, often $1 \%$ of the gyre's volume per day. This exchange is the primary driver of changes in water properties. In observations and in our model, the WAG is warmer than its surroundings and has a subsurface salinity minimum in its center, but these differences are reduced over our period of analysis, primarily due to advection. The increase in salinity occurs on weekly to monthly time scales throughout the analysis period, but the cooling is episodic and primarily occurs in February.

The Eulerian WAG is anticyclonic, and the mean relative vorticity is anticyclonic throughout most of the gyre. Diffusion of vorticity by subgrid-scale turbulence is the main support for the continued rotation of the gyre, representing driving by the adjacent AJ and the outflow toward the Strait of Gibraltar at depth. Advection of vorticity, by contrast, adds cyclonic vorticity, both in monthly means and the 148-day mean. This erosion of the Eulerian WAG through advection was previously discussed by Sánchez-Garrido et al. (2013) in the immediate lead-up to a collapse of the gyre, though no formal budgets were presented. Our work demonstrates that even 


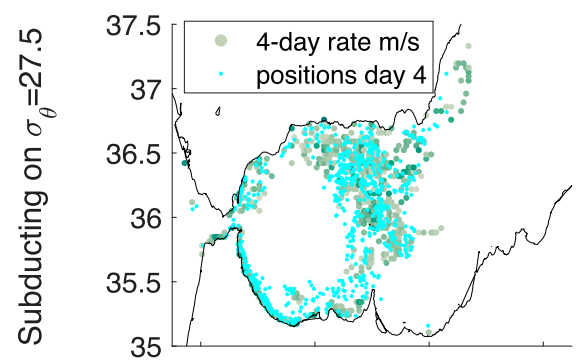

(a)

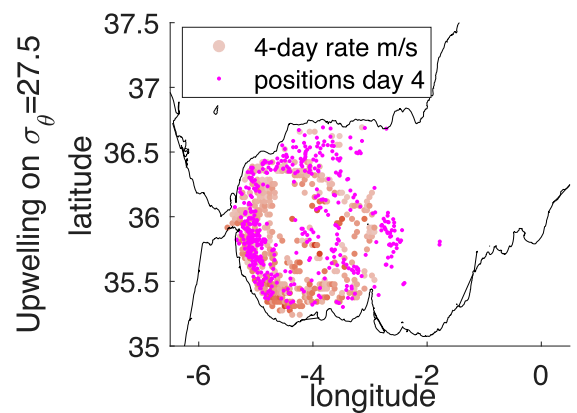

(d)

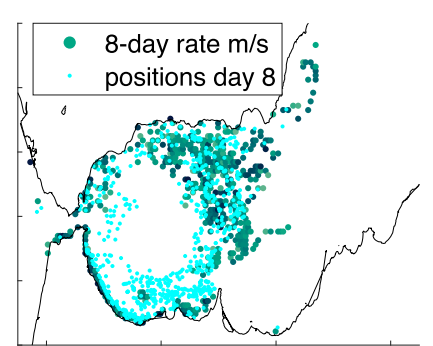

(b)

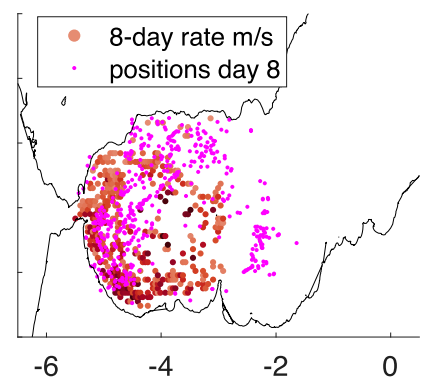

(e)

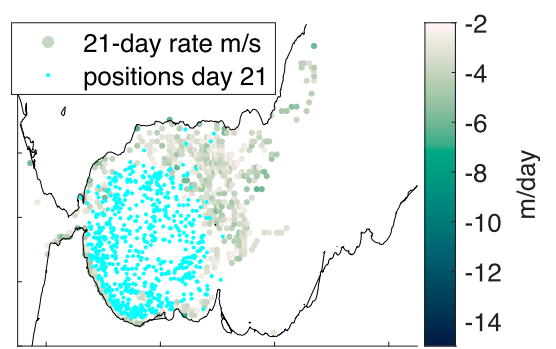

(c)

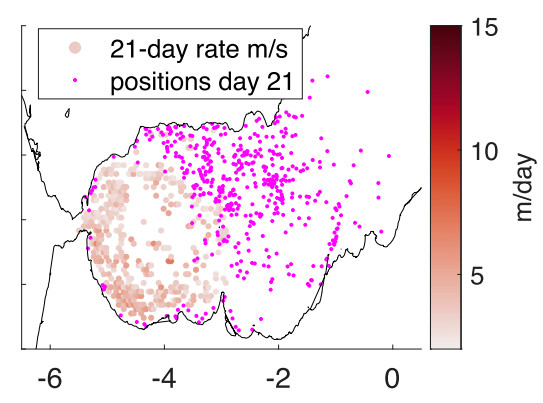

(f)

FIG. 19. (top) Lagrangian subducting trajectories on $\sigma_{\theta}=27.5 \mathrm{~kg} \mathrm{~m}^{-3}$. Initial positions colored by time-average Lagrangian vertical velocities in greens and final positions in cyan after (a) 4, (b) 8, and (c) 21 days of integration. Vertical velocities are averaged across trajectories that start in the same position from the nine releases. (d)-(f) As in (a)-(c), but for upwelling, with Lagrangian vertical velocities in reds and final positions in magenta.

while the gyre appears stable, the advective exchange between the gyre and its surroundings opposes its rotation, such that the circulation of the gyre is slightly reduced by the end of our analysis period.

To better understand the advective transport that plays such a large role in our Eulerian budgets, we performed a Lagrangian analysis of the stirring and exchange between the WAG and its surroundings. Our Lagrangian analysis describes the geometry of the WAG within the Alboran Sea. We used manifold segments to identify the finite-time stirring region near the WAG periphery, where water may be exchanged with surroundings on the time scale of 1-2 weeks. This region extends throughout much of the western Alboran Sea, from the outer regions of the gyre itself to the Strait of Gibraltar, the northwestern coastal region, and the central Alboran. In contrast, the geographical extent of the finite-time core region, where there are no turnstile lobe exchanges with surroundings at any time during the 5 months analyzed, is comparatively small, but its extent is larger at depth where the stirring region is smaller. The increase in size of the finite-time core and decrease in size of the finite-time stirring region with depth is due to the decrease in velocities.

One of the goals of our work was to compare, contrast, and reconcile the Eulerian and Lagrangian approaches to the study of the WAG. Any definition, Eulerian or Lagrangian, of a gyre boundary in a time-dependent flow is likely to be subjective. Possible Eulerian boundaries based on hydrographic or kinematic properties will tend to differ from each other, and Lagrangian boundaries depend on the exact definition and protocol for defining coherent sets or structures, and separatrices between them. Our work employed one reasonable definition of an Eulerian boundary and one reasonable choice of a Lagrangian boundary. The Eulerian budgets indicate that advection of water into the Eulerian WAG does not constantly add warm, fresh, anticyclonic water, as might be expected if the water were coming from only the Atlantic Jet. The Lagrangian analysis of the region where advective exchange can occur offers an explanation of these fluxes of cool, salty, cyclonic water: the advective exchange can reach the rest of the western Alboran Sea, not just the AJ, and add water from these other sources.

A Lagrangian budget for the evolving WAG would allow further reconciliation between the Eulerian and Lagrangian views of this region. However, there are challenges in forming such a budget that prevented us from doing so here. First, the necessary resolution of the manifolds in time and depth may be higher than the daily and six-surface manifolds we have used here. Second, the complex geometry of the manifolds creates challenges in accurately constructing an evolving lateral boundary and computing the contents within that boundary. This is especially true at times when secondary circulations and secondary hyperbolic regions are present near Ceuta and Melilla.

We also used the manifolds to identify lobes, distinct volumes of water that are exchanged between the WAG and its surroundings. One such lobe was identified and tracked over several days to demonstrate a path of water entering the gyre. As the lobe entered the WAG, it contracted horizontally and 
TABLE A1. Table of names of model grid and constants and what they contain.

\begin{tabular}{|c|c|c|}
\hline Name & Field & Units \\
\hline \multicolumn{3}{|c|}{ Grid } \\
\hline $\mathrm{rAc}$ & $\begin{array}{l}\text { Temperature, salinity, vertical velocity } \\
\text { (TSW) cell area }\end{array}$ & $\mathrm{m}^{2}$ \\
\hline $\mathrm{rAz}$ & Vorticity cell areas & $\mathrm{m}^{2}$ \\
\hline $\mathrm{drF}$ & Cell heights & $\mathrm{m}$ \\
\hline $\mathrm{hFacC}$ & Portion of TSW cell height in water & $\mathrm{m}^{2}$ \\
\hline hFacW & $\begin{array}{l}\text { Portion of western edge of TSW cell } \\
\text { height in water }\end{array}$ & $\mathrm{m}^{2}$ \\
\hline hFacS & $\begin{array}{l}\text { Portion of southern edge of TSW cell } \\
\text { height in water }\end{array}$ & $\mathrm{m}^{2}$ \\
\hline $\mathrm{dxg}$ & Zonal cell edge length & $\mathrm{m}$ \\
\hline dyg & Meridional cell edge length & $\mathrm{m}$ \\
\hline dxc & Zonal distance between TSW cell centers & $\mathrm{m}$ \\
\hline dyc & $\begin{array}{l}\text { Meridional distance between TSW cell } \\
\text { centers }\end{array}$ & $\mathrm{m}$ \\
\hline \multicolumn{3}{|c|}{ Constants from data or STDOUT } \\
\hline rhoConst & Background density & $\mathrm{kg} \mathrm{m}^{-3}$ \\
\hline $\mathrm{CP}$ & Specific heat & $\mathrm{J} \mathrm{kg}^{-1{ }^{\circ} \mathrm{C}}$ \\
\hline
\end{tabular}

extended vertically, exhibiting a Lagrangian subduction path. We then confirmed that this subduction behavior is general by showing the subducting and upwelling paths that a larger set of Lagrangian trajectories follow. These results support continued research into Lagrangian subduction and upwelling, especially in the Alboran Sea.

Overall, we have learned from our analyses that the WAG is quite well connected to the rest of the western Alboran Sea. The associated advective exchange is a dominant term in all of the water property budgets of the gyre. However, its effects on salinity, temperature and vorticity are different. For salinity alone the advective process drives a consistent cumulative erosion of the gyre's salinity minimum, while cooling occurred mainly in one month, and changes in vorticity were small. There are three avenues for future work: first, an examination of the drivers of the heaving isopycnals would explain the large contributions of vertical advection to the Eulerian budgets; second, a full Lagrangian budget for the water properties would clarify whether the Lagrangian WAG is decaying over the period when the WAG appears stable; third, a separate study of the collapse of the gyre could identify the trigger and quantify the associated processes.

Acknowledgments. This work was supported on DOD (MURI) Grant N000141110087, ONR Grant N000141812417, and NSF Grant OCE-1558806. We thank two anonymous reviewers for substantial input, Jean-Michel Campin for assistance with the MITgcm, and Dan Collura for editing support.

Data availability statement. All needed data are available: Brett (2020a) has raw output for daily $T, S$, and 3D velocities; Brett (2020c) has rotated daily and 148-day mean velocities; Brett (2020b) has all budget terms, manifolds, and their frequency maps. Code for creating manifolds and Eulerian budgets are available as well from Brett (2020d).

\section{APPENDIX}

\section{Numerical Budget Computations}

The MITgcm may be configured to save out all terms necessary to close the budgets for volume, salinity, temperature, and momentum. Details for computing salinity and temperature budgets are provided in Chakraborty and Campin (2014). In this appendix, we provide the relevant information to compute the vertical vorticity budget. Table A1 lists the set of grid information and model constants that are needed for understanding the budgets. Table A2 lists the diagnostics needed to close the vertical vorticity budget. Additional examples are available in Brett (2018, appendix B).

The numerical vertical vorticity budget is the curl of the horizontal momentum budgets and is defined at the corners of temperature-salinity-vertical velocity (TSW) cells. For a budget integrated over some area of vorticity cells, Stokes' theorem means one can integrate the momentum components around the edge of cells rather than integrating the curl over the area.

Numerical budgets for horizontal momentum, in terms representing standard physical processes, can be written

$$
\begin{aligned}
U \_t \text { tend }= & \text { Um_Advec }+ \text { UPress_tend }+ \text { UDif_tend }+ \text { UDiss } \\
& + \text { Um_Ext }, \\
V \_ \text {tend }= & \text { Vm_Advec }+ \text { VPress_tend }+ \text { VDif_tend }+ \text { VDiss } \\
& + \text { Vm_Ext },
\end{aligned}
$$

where $U$ indicates the grid-aligned meridional and $V$ the gridaligned zonal velocity. The advection terms, _Advec, include Coriolis effects; _Cori is available for separation. The diffusion terms are vertical diffusion UDif_tend and dissipation UDiss, which includes horizontal diffusion as well as bottom and side drag. Bottom and side drag are available for separation, e.g., USidDrag. The first, fourth, and last terms on the right hand side are diagnostics. The _tend terms are calculated from diagnostics as follows:

$$
\begin{aligned}
& U \_ \text {tend }=\text { TOTUTEND } / 86400 \text {, } \\
& V \_ \text {tend }=\text { TOTVTEND } / 86400 \text {, } \\
& \text { UPress_tend }=\mathrm{Um} \_\mathrm{dPHdx}-g[\operatorname{SSH}(x i+1, y i) \\
& -\operatorname{SSH}(x i, y i)] / \mathrm{dxc} \text {, } \\
& \text { VPress_tend }=\text { Vm_dPHdy }-g[\operatorname{SSH}(x i, y i+1) \\
& -\operatorname{SSH}(x i, y i)] / d y c \text {, } \\
& \text { UDif_tend }=\text { [VISrI_Um }(x i, y i, z i+1) \\
& \text {-VISrI_Um }(x i, y i, z i)] /(\mathrm{rAw} \times \mathrm{drF} \times \mathrm{hFacW}) \text {, } \\
& \text { VDif_tend }=\text { [VISrI_Vm }(x i, y i, z i+1) \\
& \text {-VISrI_Vm }(x i, y i, z i)] /(\mathrm{rAs} \times \mathrm{drF} \times \mathrm{hFacS}) \text {. }
\end{aligned}
$$

All zonal terms are defined at the center of the western edge of TSW cells. All meridional terms are defined at the center of the southern edge of TSW cells. 
TABLE A2. Table of diagnostic names and what they contain. These diagnostics can close the horizontal momentum and vertical vorticity budgets.

\begin{tabular}{|c|c|c|}
\hline Name & Field & Units \\
\hline $\begin{array}{l}\text { TOTUTEND } \\
\text { TOTVTEND }\end{array}$ & $\begin{array}{l}\text { Change in time } \\
\text { Total zonal velocity tendency } \\
\text { Total meridional velocity tendency }\end{array}$ & $\begin{array}{l}\mathrm{m} \mathrm{s}^{-1} \text { day } \\
\mathrm{m} \mathrm{s}^{-1} \text { day }\end{array}$ \\
\hline $\begin{array}{l}\text { Um_Advec } \\
\text { Vm_Advec }\end{array}$ & $\begin{array}{l}\qquad \text { Advection } \\
\text { Zonal velocity tendency due to advection of momentum } \\
\text { Meridional velocity tendency due to advection of momentum }\end{array}$ & $\begin{array}{l}\mathrm{m} \mathrm{s}^{-2} \\
\mathrm{~m} \mathrm{~s}^{-2}\end{array}$ \\
\hline $\begin{array}{l}\text { VISrI_Um } \\
\text { VISrI_Vm } \\
\text { Um_Diss } \\
\text { Vm_Diss } \\
\text { UBotDrag } \\
\text { VBotDrag } \\
\text { USidDrag } \\
\text { VSidDrag }\end{array}$ & $\begin{array}{l}\quad \text { Mixing } \\
\text { Vertical implicit zonal momentum diffusion } \\
\text { Vertical implicit meridional momentum diffusion } \\
\text { Horizontal diffusion and drag of zonal momentum } \\
\text { Horizontal diffusion and drag of meridional momentum } \\
\text { Bottom drag of zonal momentum } \\
\text { Bottom drag of meridional momentum } \\
\text { Side drag of zonal momentum } \\
\text { Side drag of meridional momentum }\end{array}$ & $\begin{array}{l}\mathrm{m}^{4} \mathrm{~s}^{-2} \\
\mathrm{~m}^{4} \mathrm{~s}^{-2} \\
\mathrm{~m} \mathrm{~s}^{-2} \\
\mathrm{~m} \mathrm{~s}^{-2} \\
\mathrm{~m} \mathrm{~s}^{-2} \\
\mathrm{~m} \mathrm{~s}^{-2} \\
\mathrm{~m} \mathrm{~s}^{-2} \\
\mathrm{~m} \mathrm{~s}^{-2}\end{array}$ \\
\hline $\begin{array}{l}\text { Um_Ext } \\
\text { Vm_Ext }\end{array}$ & $\begin{array}{l}\quad \text { Forcing } \\
\text { Zonal velocity tendency due to wind } \\
\text { Meridional velocity tendency due to wind }\end{array}$ & $\begin{array}{l}\mathrm{m} \mathrm{s}^{-2} \\
\mathrm{~m} \mathrm{~s}^{-2}\end{array}$ \\
\hline $\begin{array}{l}\text { Um_Cori } \\
\text { Vm_Cori } \\
\text { Um_dPHdx } \\
\text { Vm_dPHdy } \\
\text { momVort3 }\end{array}$ & $\begin{array}{l}\text { Other } \\
\text { Zonal velocity tendency due to Coriolis term } \\
\text { Meridional velocity tendency due to Coriolis term } \\
\text { Zonal velocity tendency due to pressure gradient term } \\
\text { Meridional velocity tendency due to pressure gradient term } \\
\text { Vertical component of vorticity }\end{array}$ & $\begin{array}{l}\mathrm{m} \mathrm{s}^{-2} \\
\mathrm{~m} \mathrm{~s}^{-2} \\
\mathrm{~m} \mathrm{~s}^{-2} \\
\mathrm{~m} \mathrm{~s}^{-2} \\
\mathrm{~s}^{-1}\end{array}$ \\
\hline
\end{tabular}

Forming the vertical relative vorticity budget requires using Stokes' Theorem on each horizontal layer, being certain that only wet edges are used (where hFacS and hFacW are one). For each layer of the volume, identify the edges of the area to be integrated over, defining open $\mathrm{N}$ for northern edges, openE for eastern, openW for western, and openS for southern. Each term in the vorticity budget matching those in the momentum budgets can be calculated like

$$
\begin{aligned}
\zeta_{-} \text {tend }=- & U_{-} \text {tend }(\text { openN }) \times \mathrm{dxc}(\text { openN })+U_{-} \text {tend }(\text { openS }) \\
& \times \mathrm{dxc}(\text { openS })+V_{-} \text {tend }(\text { openE }) \times \mathrm{dyc}(\text { openE }) \\
& -V_{-} \text {tend }(\text { open } \mathrm{W}) \times \mathrm{dxc}(\text { open } \mathrm{W})
\end{aligned}
$$

Then the vertical vorticity numerical budget will have terms

$$
\zeta_{-} \text {tend }=\zeta_{-} \text {Advec }+\zeta_{-} \text {Press }+\zeta_{-} \text {Dif_tend }+\zeta_{-} \text {Diss }+\zeta \text { Ext. }
$$

Note that the pressure term is calculated using Um_dPHdx and Vm_dPHdy, giving the baroclinic pressure effect, $\left(-1 / \rho^{2}\right)(\nabla P \times$ $\nabla \rho)$ in the physical equation. The calculation has been described for a single layer. For our vertical integral, we multiply the terms from each layer by $\mathrm{drF}$ before summing.

\section{REFERENCES}

Bormans, M., and C. Garrett, 1989: A simple criterion for gyre formation by the surface outflow from a strait, with application to the Alboran Sea.J. Geophys. Res., 94, 12 637-12 644, https:// doi.org/10.1029/JC094iC09p12637.
Branicki, M., and A. Kirwan Jr., 2010: Stirring: The Eckart paradigm revisited. Int. J. Eng. Sci., 48, 1027-1042, https://doi.org/ 10.1016/j.ijengsci.2010.08.003.

Brett, G., 2018: Chaotic advection, mixing, and property exchange in three-dimensional ocean eddies and gyres. Ph.D. thesis, Massachusetts Institute of Technology, 268 pp.

, 2020a: MITgcm Alboran Sea 2007-2008 TSUVW. Zenodo, https://doi.org/10.5281/zenodo.3626166.

_ 2020b: MITgcm Alboran Sea budgets and manifolds Nov 2007Mar 2008. Zenodo, https://doi.org/10.5281/zenodo.3628892.

—, 2020c: MITgcm Alboran Sea velocities 2007-2008. Zenodo, https://doi.org/10.5281/zenodo.3614122.

— 2020d: Western Alboran Gyre code and figures (v1.0.0). Zenodo, https://doi.org/10.5281/zenodo.3633361.

Bryden, H. L., and H. M. Stommel, 1982: Origin of the Mediterranean outflow. J. Mar. Res., 40, 55-71.

Chakraborty, A., and J.-M. Campin, 2014: Heat and salt budget in MITgcm. Tech. Doc., 7 pp., http://mitgcm.org/download/daily_ snapshot/MITgcm/doc/Heat_Salt_Budget_MITgcm.pdf.

Coulliette, C., and S. Wiggins, 2000: Intergyre transport in a winddriven, quasigeostrophic double gyre: An application of lobe dynamics. Nonlinear Processes Geophys., 7, 59-85, https:// doi.org/10.5194/npg-7-59-2000.

Deese, H. E., L. J. Pratt, and K. R. Helfrich, 2002: A laboratory model of exchange and mixing between western boundary layers and subbasin recirculation gyres. J. Phys. Oceanogr., 32, 1870-1889, https://doi.org/10.1175/1520-0485(2002)032<1870: ALMOEA $>2.0 . \mathrm{CO} ; 2$.

Duan, J., and S. Wiggins, 1996: Fluid exchange across a meandering jet quasiperiodic variability. J. Phys. Oceanogr., 26, 1176-1188, 
http://doi.org/10.1175/1520-0485(1996)026\%3C1176:FEAAMJ \%3E2.0.CO;2.

Echevarría, F., L. Zabala, A. Corzo, G. Navarro, L. Prieto, and D. Macías, 2009: Spatial distribution of autotrophic picoplankton in relation to physical forcings: The Gulf of Cádiz, Strait of Gibraltar and Alborán Sea case study. J. Plankton Res., 31, 1339-1351, https://doi.org/10.1093/plankt/fbp070.

Flexas, M. M., D. Gomis, S. Ruiz, A. Pascual, and P. León, 2006: In situ and satellite observations of the eastward migration of the western Alboran Sea gyre. Prog. Oceanogr., 70, 486-509, https://doi.org/10.1016/j.pocean.2006.03.017.

Hadjighasem, A., M. Farazmand, D. Blazevski, G. Froyland, and G. Haller, 2017: A critical comparison of Lagrangian methods for coherent structure detection. Chaos Interdiscip. J. Nonlinear Sci., 27, 053104, http://doi.org/10.1063/1.4982720.

Haller, G., 2015: Lagrangian coherent structures. Annu. Rev. Fluid Mech., 47, 137-162, https://doi.org/10.1146/annurevfluid-010313-141322.

—_, and A. Poje, 1998: Finite time transport in aperiodic flows. Physica D, 119, 352-380, https://doi.org/10.1016/S01672789(98)00091-8.

— barriers in two-dimensional flows. Physica D, 241, 1680-1702, https://doi.org/10.1016/j.physd.2012.06.012.

_- A. Hadjighasem, M. Farazmand, and F. Huhn, 2016: Defining coherent vortices objectively from the vorticity. J. Fluid Mech., 795, 136-173, https://doi.org/10.1017/jfm.2016.151.

—, D. Karrasch, and F. Kogelbauer, 2018: Material barriers to diffusive and stochastic transport. Proc. Natl. Acad. Sci. USA, 115, 9074-9079, https://doi.org/10.1073/pnas.1720177115.

Heburn, G. W., and P. E. La Violette, 1990: Variations in the structure of the anticyclonic gyres found in the Alboran Sea. J. Geophys. Res., 95, 1599-1613, https://doi.org/10.1029/ JC095iC02p01599.

Large, W. G., J. C. McWilliams, and S. C. Doney, 1994: Oceanic vertical mixing: A review and a model with a nonlocal boundary layer parameterization. Rev. Geophys., 32, 363-403, https://doi.org/10.1029/94RG01872.

Mancho, A. M., D. Small, S. Wiggins, and K. Ide, 2003: Computation of stable and unstable manifolds of hyperbolic trajectories in twodimensional, aperiodically time-dependent vector fields. Physica $D, \mathbf{1 8 2}, 188-222$, https://doi.org/10.1016/S0167-2789(03)00152-0.

Marshall, J., A. Adcroft, C. Hill, L. Perelman, and C. Heisey, 1997: A finite-volume, incompressible Navier Stokes model for studies of the ocean on parallel computers. J. Geophys. Res., 102, 5753-5766, https://doi.org/10.1029/96JC02775.

Miller, P. D., L. J. Pratt, K. R. Helfrich, and C. K. Jones, 2002: Chaotic transport of mass and potential vorticity for an island recirculation. J. Phys. Oceanogr., 32, 80-102, https://doi.org/ 10.1175/1520-0485(2002)032<0080:CTOMAP > 2.0.CO;2.

Peliz, A., D. Boutov, and A. Teles-Machado, 2013: The Alboran Sea mesoscale in a long term high resolution simulation: Statistical analysis. Ocean Modell., 72, 32-52, https://doi.org/ 10.1016/j.ocemod.2013.07.002.

Preller, R. H., 1986: A numerical model study of the Alboran Sea gyre. Prog. Oceanogr., 16, 113-146, https://doi.org/10.1016/ 0079-6611(86)90031-5.
Renault, L., T. Oguz, A. Pascual, G. Vizoso, and J. Tintoré, 2012: Surface circulation in the Alborán Sea (western Mediterranean) inferred from remotely sensed data. J. Geophys. Res., 117, n/a, https://doi.org/10.1029/2011JC007659.

Rypina, I. I., M. G. Brown, and H. Koçak, 2009: Transport in an idealized three-gyre system with application to the Adriatic Sea. J. Phys. Oceanogr., 39, 675-690, https://doi.org/10.1175/ 2008JPO3975.1.

— L. J. Pratt, J. Pullen, J. Levin, and A. L. Gordon, 2010: Chaotic advection in an archipelago. J. Phys. Oceanogr., 40, 1988-2006, https://doi.org/10.1175/2010JPO4336.1.

Samelson, R. M., 1992: Fluid exchange across a meandering jet. J. Phys. Oceanogr., 22, 431-444, https://doi.org/10.1175/15200485(1992)022<0431:FEAAMJ > 2.0.CO;2.

and Waves: The Dynamical Systems Approach. Interdisciplinary Applied Mathematics Series, Vol. 31, Springer, 150 pp., https:// doi.org/10.1007/978-0-387-46213-4.

Sammartino, S., J. García Lafuente, C. Naranjo, J. C. Sánchez Garrido, R. Sánchez Leal, and A. Sánchez Román, 2015: Ten years of marine current measurements in Espartel Sill, Strait of Gibraltar. J. Geophys. Res. Oceans, 120, 6309-6328, https:// doi.org/10.1002/2014JC010674.

Sánchez-Garrido, J. C., J. G. Lafuente, E. Á. Fanjul, M. G. Sotillo, and J. Francisco, 2013: What does cause the collapse of the western Alboran gyre? Results of an operational ocean model. Prog. Oceanogr., 116, 142-153, https://doi.org/ 10.1016/j.pocean.2013.07.002.

Sarhan, T., J. G. Lafuente, M. Vargas, J. M. Vargas, and F. Plaza, 2000: Upwelling mechanisms in the northwestern Alboran Sea. J. Mar. Syst., 23, 317-331, https://doi.org/10.1016/S09247963(99)00068-8.

Sayol, J.-M., A. Orfila, G. Simarro, C. López, L. Renault, A. Galán, and D. Conti, 2013: Sea surface transport in the western Mediterranean Sea: A Lagrangian perspective. J. Geophys. Res. Oceans, 118, 6371-6384, https://doi.org/10.1002/2013JC009243.

Vargas-Yáñez, M., F. Plaza, J. Garcia-Lafuente, T. Sarhan, J. Vargas, and P. Vélez-Belchí, 2002: About the seasonal variability of the Alboran Sea circulation. J. Mar. Syst., 35, 229-248, https:// doi.org/10.1016/S0924-7963(02)00128-8.

Viúdez, Á., J. Tintoré, and R. L. Haney, 1996: Circulation in the Alboran Sea as determined by quasi-synoptic hydrographic observations. Part I: Three-dimensional structure of the two anticyclonic gyres. J. Phys. Oceanogr., 26, 684-705, https:// doi.org/10.1175/1520-0485(1996)026<0684: CITASA $>2.0 . \mathrm{CO} ; 2$.

_ J.-M. Pinot, and R. L. Haney, 1998: On the upper layer circulation in the Alboran Sea. J. Geophys. Res., 103, $21653-$ 21 666, https://doi.org/10.1029/98JC01082.

Whitehead, J. A., Jr., and A. Miller, 1979: Laboratory simulation of the gyre in the Alboran Sea. J. Geophys. Res., 84, 3733-3742, https://doi.org/10.1029/JC084iC07p03733.

Yuan, G., L. Pratt, and C. Jones, 2004: Cross-jet Lagrangian transport and mixing in a $21 / 2$-layer model. J. Phys. Oceanogr., 34, 1991-2005, https://doi.org/10.1175/1520-0485(2004)034<1991: CLTAMI $>2.0 . \mathrm{CO} ; 2$. 